Supplementary material belonging to the publication:

\title{
1,1,2,2-Tetramethyl-1,2-bis(phenylthiomethyl)disilane, a Flexible Ligand
} for the Construction of Macrocyclic, Mesocyclic and Bridged Dithioether Complexes. Synthesis of the Bis-silylated Olefins Z$\left(\mathrm{PhSCH}_{2}\right) \mathrm{Me}_{2} \mathrm{SiC}(\mathrm{H})=\mathrm{C}(\mathrm{Ar}) \mathrm{SiMe}_{2}\left(\mathrm{CH}_{2} \mathrm{SPh}\right)$ by Catalytic Activation of the Si-Si Bond.

Harmel N. Peindy ${ }^{a}$, Fabrice Guyon ${ }^{a}$, Isabelle Jourdain ${ }^{a}$, Michael Knorr ${ }^{a *}$, Daniel Schildbach $^{\mathrm{b}}$ and Carsten Strohmann ${ }^{\mathrm{b} *}$

a'Laboratoire de Chimie des Matériaux et Interfaces, Université de Franche-Comté , Faculté des Sciences et des Techniques, 16, Route de Gray, 25030 Besançon Cedex, France.

E-mail: michael.knorr@univ-fcomte.fr

${ }^{b}$ Institut für Anorganische Chemie der Universität Würzburg, Am Hubland, D-97074, Würzburg, Germany

E-mail: mail@carsten-strohmann.de

Table 1. Crystal data and structure refinement for $\mathbf{2 a}$.

Table 2. Atomic coordinates $\left(\times 10^{4}\right)$ and equivalent isotropic displacement parameters $\left(\AA^{2} \times 10^{-1}\right)$ for $\mathbf{2 a}$.

Table 3. Bond lengths $[\AA]$ and angles $\left[^{\circ}\right]$ for $\mathbf{2 a}$.

Table 4. Anisotropic displacement parameters $\left(\AA^{2} \times 10^{-1}\right)$ for $\mathbf{2 a}$.

Table 5. Hydrogen coordinates $\left(\times 10^{4}\right)$ and isotropic displacement parameters $\left(\AA^{2} \times 10^{-1}\right)$ for $2 \mathbf{a}$.

ORTEP Plot for $\mathbf{2 a}$.

Table 6. Crystal data and structure refinement for $\mathbf{2 b}$.

Table 7. Atomic coordinates $\left(\times 10^{4}\right)$ and equivalent isotropic displacement parameters $\left(\AA^{2} \times 10^{-1}\right)$ for $\mathbf{2 b}$.

Table $8 . \quad$ Bond lengths $[\AA]$ and angles $\left[{ }^{\circ}\right]$ for $\mathbf{2 b}$. 
Table 9. Anisotropic displacement parameters $\left(\AA^{2} \times 10^{-1}\right)$ for $\mathbf{2 b}$.

Table 10. Hydrogen coordinates $\left(\times 10^{4}\right)$ and isotropic displacement parameters $\left(\AA^{2} \times 10^{-1}\right)$ for $\mathbf{2 b}$.

ORTEP Plot for $\mathbf{2 b}$.

Table 11. Crystal data and structure refinement for 4 .

Table 12. Atomic coordinates $\left(\times 10^{4}\right)$ and equivalent isotropic displacement parameters $\left(\AA^{2} \times 10^{-1}\right)$ for 4 .

Table 13. Bond lengths $[\AA]$ and angles $\left[{ }^{\circ}\right]$ for 4.

Table 14. Anisotropic displacement parameters $\left(\AA^{2} \times 10^{-1}\right)$ for 4 .

Table 15. Hydrogen coordinates $\left(\times 10^{4}\right)$ and isotropic displacement parameters $\left(\AA^{2} \times 10^{-1}\right)$ for 4 .

ORTEP Plot for 4.

Table 16. Crystal data and structure refinement for $\mathbf{5}$.

Table 17. Atomic coordinates $\left(\times 10^{4}\right)$ and equivalent isotropic displacement parameters $\left(\AA^{2} \times 10^{-1}\right)$ for 5 .

Table 18. Bond lengths $[\AA]$ and angles $\left[^{\circ}\right]$ for 5 .

Table 19. Anisotropic displacement parameters $\left(\AA^{2} \times 10^{-1}\right)$ for $\mathbf{5}$.

Table 20. Hydrogen coordinates $\left(\times 10^{4}\right)$ and isotropic displacement parameters $\left(\AA^{2} \times 10^{-1}\right)$ for 5 .

ORTEP Plot for 5.

Table 21. Crystal data and structure refinement for $\mathbf{6}$.

Table 22. Atomic coordinates $\left(\times 10^{4}\right)$ and equivalent isotropic displacement parameters $\left(\AA^{2} \times 10^{-1}\right)$ for 6 .

Table 23. Bond lengths $[\AA]$ and angles $\left[{ }^{\circ}\right]$ for 6 .

Table 24. Anisotropic displacement parameters $\left(\AA^{2} \times 10^{-1}\right)$ for 6 .

Table 25. Hydrogen coordinates $\left(\times 10^{4}\right)$ and isotropic displacement parameters $\left(\AA^{2} \times 10^{-1}\right)$ for 6 .

ORTEP Plot for 6 .

Table 26. Crystal data and structure refinement for 7.

Table 27. Atomic coordinates $\left(\times 10^{4}\right)$ and equivalent isotropic displacement parameters $\left(\AA^{2} \times 10^{-1}\right)$ for 7 . 
Table 28. Bond lengths $[\AA]$ and angles $\left[{ }^{\circ}\right]$ for 7 .

Table 29. Anisotropic displacement parameters $\left(\AA^{2} \times 10^{-1}\right)$ for 7 .

Table 30. Hydrogen coordinates $\left(\times 10^{4}\right)$ and isotropic displacement parameters $\left(\AA^{2} \times 10^{-1}\right)$ for 7.

ORTEP Plot for 7.

Absorption spectra of 1 and $2 \mathrm{a}$ carried out in $\mathrm{CH}_{2} \mathrm{Cl}_{2}$ at $298 \mathrm{~K}$.

${ }^{195} \mathrm{Pt}\left\{{ }^{1} \mathrm{H}\right\}$ NMR spectrum of $\mathbf{2 a}$ in $\mathrm{CDCl}_{3}$ recorded at 298 and $323 \mathrm{~K}$. 
Table 1. Crystal data and structure refinement for $\mathbf{2 a}$.

Identification code

Empirical formula

Formula weight

Temperature

Wavelength

Crystal system

Space group

Unit cell dimensions

Volume

Z

Density (calculated)

Absorption coefficient

$\mathrm{F}(000)$

Crystal size

Theta range for data collection

Index ranges

Reflections collected

Independent reflections

Completeness to theta $=26.00^{\circ}$

Max. and min. transmission

Refinement method

Data / restraints / parameters

Goodness-of-fit on $\mathrm{F}^{2}$

Final $\mathrm{R}$ indices $[\mathrm{I}>2 \operatorname{sigma}(\mathrm{I})]$

$\mathrm{R}$ indices (all data)

Largest diff. peak and hole 2a

C18 H26 C12 Pt S2 Si2

628.68

173(2) K

$0.71073 \AA$

triclinic

P-1

$$
\begin{array}{ll}
\mathrm{a}=7.472(2) \AA & \alpha=98.43(3)^{\circ} . \\
\mathrm{b}=9.403(2) \AA & \beta=91.64(4)^{\circ} . \\
\mathrm{c}=17.115(5) \AA & \gamma=104.07(3)^{\circ} .
\end{array}
$$

1151.3(5) $\AA^{3}$

2

$1.814 \mathrm{Mg} / \mathrm{m}^{3}$

$6.612 \mathrm{~mm}^{-1}$

612

$0.30 \times 0.20 \times 0.20 \mathrm{~mm}^{3}$

2.26 to $26.00^{\circ}$

$-9<=\mathrm{h}<=9,-11<=\mathrm{k}<=11,-21<=\mathrm{l}<=21$

13259

$4243[\mathrm{R}($ int $)=0.0824]$

$93.8 \%$

0.3514 and 0.2417

Full-matrix least-squares on $\mathrm{F}^{2}$

4243 / 0 / 270

1.054

$\mathrm{R} 1=0.0570, \mathrm{wR} 2=0.1521$

$\mathrm{R} 1=0.0589, \mathrm{wR} 2=0.1539$

4.310 and -4.990 e. $\AA^{-3}$ 
Table 2. Atomic coordinates ( $\left.\times 10^{4}\right)$ and equivalent isotropic displacement parameters $\left(\AA^{2} \times 10^{3}\right)$ for 2 a. $U(e q)$ is defined as one third of the trace of the orthogonalized $U^{i j}$ tensor.

\begin{tabular}{|c|c|c|c|c|}
\hline & $\mathrm{x}$ & $\mathrm{y}$ & z & $\mathrm{U}(\mathrm{eq})$ \\
\hline $\mathrm{C}(1)$ & $2857(12)$ & $3565(11)$ & $2358(5)$ & $22(2)$ \\
\hline$C(2)$ & $1135(12)$ & $-43(11)$ & $3422(5)$ & $26(2)$ \\
\hline$C(3)$ & $1793(16)$ & $5251(14)$ & $3812(6)$ & $36(2)$ \\
\hline$C(4)$ & $5710(15)$ & $4778(13)$ & $3764(6)$ & $36(2)$ \\
\hline$C(5)$ & 261(19) & $2106(15)$ & $4741(7)$ & $47(3)$ \\
\hline$C(6)$ & 4139(19) & $1559(14)$ & $4716(7)$ & $44(3)$ \\
\hline$C(7)$ & $3764(12)$ & $2226(11)$ & $885(5)$ & $24(2)$ \\
\hline$C(8 \mathrm{~A})$ & $4280(30)$ & $1000(30)$ & $464(12)$ & $38(5)$ \\
\hline $\mathrm{C}(8 \mathrm{~B})$ & $4790(30)$ & $3160(30)$ & $380(12)$ & $36(5)$ \\
\hline$C(9 A)$ & $3750(40)$ & $580(30)$ & $-355(14)$ & $49(6)$ \\
\hline$C(9 B)$ & $4290(30)$ & $2860(40)$ & $-441(15)$ & $58(9)$ \\
\hline$C(10)$ & 2651(19) & $1488(19)$ & $-721(7)$ & $52(4)$ \\
\hline$C(11 \mathrm{~A})$ & $2100(40)$ & $2430(30)$ & $-258(14)$ & $44(6)$ \\
\hline$C(11 B)$ & $1790(30)$ & $930(30)$ & $-225(14)$ & $41(5)$ \\
\hline$C(12 A)$ & $2620(40)$ & $2880(30)$ & $530(13)$ & $42(5)$ \\
\hline$C(12 B)$ & $2250(30)$ & $1250(30)$ & $585(13)$ & $41(5)$ \\
\hline$C(13)$ & $1098(13)$ & $-1985(11)$ & $1989(5)$ & $28(2)$ \\
\hline$C(14)$ & $-334(14)$ & $-2985(12)$ & $2292(7)$ & $37(2)$ \\
\hline$C(15)$ & $-924(17)$ & $-4423(13)$ & $1912(8)$ & $46(3)$ \\
\hline$C(16)$ & $-170(30)$ & $-4870(16)$ & $1265(9)$ & $81(6)$ \\
\hline$C(17)$ & $1250(40)$ & $-3919(19)$ & $945(10)$ & $117(11)$ \\
\hline$C(18)$ & $1890(30)$ & $-2433(14)$ & $1326(8)$ & $75(6)$ \\
\hline $\mathrm{Cl}(1)$ & $8051(3)$ & $1705(3)$ & $2472(2)$ & $33(1)$ \\
\hline $\mathrm{Cl}(2)$ & $5327(4)$ & $-1252(3)$ & $2988(2)$ & $33(1)$ \\
\hline $\mathrm{Pt}$ & $4877(1)$ & $750(1)$ & $2440(1)$ & $16(1)$ \\
\hline $\mathrm{S}(1)$ & $4633(3)$ & $2788(3)$ & $1910(1)$ & $20(1)$ \\
\hline$S(2)$ & $1757(3)$ & $-67(3)$ & $2413(1)$ & $20(1)$ \\
\hline $\operatorname{Si}(1)$ & $3196(4)$ & $3924(3)$ & $3486(1)$ & $24(1)$ \\
\hline $\operatorname{Si}(2)$ & $2184(4)$ & $1812(3)$ & $4102(2)$ & $26(1)$ \\
\hline
\end{tabular}


Table 3. Bond lengths $[\AA]$ and angles $\left[^{\circ}\right]$ for $\mathbf{2 a}$.

\begin{tabular}{|c|c|c|c|}
\hline $\mathrm{C}(1)-\mathrm{S}(1)$ & $1.803(9)$ & $\mathrm{C}(12 \mathrm{~B})-\mathrm{C}(7)-\mathrm{C}(12 \mathrm{~A})$ & $69.2(16)$ \\
\hline $\mathrm{C}(1)-\mathrm{Si}(1)$ & $1.909(9)$ & $\mathrm{C}(12 \mathrm{~B})-\mathrm{C}(7)-\mathrm{C}(8 \mathrm{~A})$ & $72.3(16)$ \\
\hline$C(2)-S(2)$ & $1.800(9)$ & $\mathrm{C}(12 \mathrm{~A})-\mathrm{C}(7)-\mathrm{C}(8 \mathrm{~A})$ & $119.8(15)$ \\
\hline$C(2)-\operatorname{Si}(2)$ & $1.923(11)$ & $\mathrm{C}(12 \mathrm{~B})-\mathrm{C}(7)-\mathrm{C}(8 \mathrm{~B})$ & $118.2(14)$ \\
\hline $\mathrm{C}(3)-\mathrm{Si}(1)$ & $1.852(12)$ & $\mathrm{C}(12 \mathrm{~A})-\mathrm{C}(7)-\mathrm{C}(8 \mathrm{~B})$ & $70.8(14)$ \\
\hline $\mathrm{C}(4)-\mathrm{Si}(1)$ & $1.872(11)$ & $\mathrm{C}(8 \mathrm{~A})-\mathrm{C}(7)-\mathrm{C}(8 \mathrm{~B})$ & $89.8(15)$ \\
\hline$C(5)-\operatorname{Si}(2)$ & $1.879(11)$ & $\mathrm{C}(12 \mathrm{~B})-\mathrm{C}(7)-\mathrm{S}(1)$ & $129.0(11)$ \\
\hline$C(6)-\operatorname{Si}(2)$ & $1.857(12)$ & $C(12 A)-C(7)-S(1)$ & $123.5(13)$ \\
\hline$C(7)-C(12 B)$ & $1.31(2)$ & $\mathrm{C}(8 \mathrm{~A})-\mathrm{C}(7)-\mathrm{S}(1)$ & $116.7(10)$ \\
\hline$C(7)-C(12 A)$ & $1.35(2)$ & $\mathrm{C}(8 \mathrm{~B})-\mathrm{C}(7)-\mathrm{S}(1)$ & $112.1(10)$ \\
\hline $\mathrm{C}(7)-\mathrm{C}(8 \mathrm{~A})$ & $1.41(3)$ & $C(7)-C(8 A)-C(9 A)$ & $118.9(19)$ \\
\hline $\mathrm{C}(7)-\mathrm{C}(8 \mathrm{~B})$ & $1.43(2)$ & $\mathrm{C}(9 \mathrm{~B})-\mathrm{C}(8 \mathrm{~B})-\mathrm{C}(7)$ & $119(2)$ \\
\hline $\mathrm{C}(7)-\mathrm{S}(1)$ & $1.808(9)$ & $\mathrm{C}(8 \mathrm{~A})-\mathrm{C}(9 \mathrm{~A})-\mathrm{C}(10)$ & $117(2)$ \\
\hline $\mathrm{C}(8 \mathrm{~A})-\mathrm{C}(9 \mathrm{~A})$ & $1.42(3)$ & $\mathrm{C}(8 \mathrm{~B})-\mathrm{C}(9 \mathrm{~B})-\mathrm{C}(10)$ & $116(2)$ \\
\hline $\mathrm{C}(8 \mathrm{~B})-\mathrm{C}(9 \mathrm{~B})$ & $1.41(3)$ & $\mathrm{C}(11 \mathrm{~B})-\mathrm{C}(10)-\mathrm{C}(11 \mathrm{~A})$ & $68.9(18)$ \\
\hline $\mathrm{C}(9 \mathrm{~A})-\mathrm{C}(10)$ & $1.50(3)$ & $\mathrm{C}(11 \mathrm{~B})-\mathrm{C}(10)-\mathrm{C}(9 \mathrm{~A})$ & $71.5(17)$ \\
\hline $\mathrm{C}(9 \mathrm{~B})-\mathrm{C}(10)$ & $1.55(4)$ & $C(11 A)-C(10)-C(9 A)$ & $117.0(16)$ \\
\hline $\mathrm{C}(10)-\mathrm{C}(11 \mathrm{~B})$ & $1.19(3)$ & $\mathrm{C}(11 \mathrm{~B})-\mathrm{C}(10)-\mathrm{C}(9 \mathrm{~B})$ & $117.3(17)$ \\
\hline$C(10)-C(11 A)$ & $1.25(3)$ & $\mathrm{C}(11 \mathrm{~A})-\mathrm{C}(10)-\mathrm{C}(9 \mathrm{~B})$ & $70.5(18)$ \\
\hline$C(11 A)-C(12 A)$ & $1.37(3)$ & $\mathrm{C}(9 \mathrm{~A})-\mathrm{C}(10)-\mathrm{C}(9 \mathrm{~B})$ & $87.5(17)$ \\
\hline $\mathrm{C}(11 \mathrm{~A})-\mathrm{C}(12 \mathrm{~B})$ & $1.96(3)$ & $\mathrm{C}(10)-\mathrm{C}(11 \mathrm{~A})-\mathrm{C}(12 \mathrm{~A})$ & $127(2)$ \\
\hline $\mathrm{C}(11 \mathrm{~B})-\mathrm{C}(12 \mathrm{~B})$ & $1.39(3)$ & $\mathrm{C}(10)-\mathrm{C}(11 \mathrm{~A})-\mathrm{C}(12 \mathrm{~B})$ & $88.4(16)$ \\
\hline$C(13)-C(18)$ & $1.356(18)$ & $\mathrm{C}(12 \mathrm{~A})-\mathrm{C}(11 \mathrm{~A})-\mathrm{C}(12 \mathrm{~B})$ & $50.0(15)$ \\
\hline$C(13)-C(14)$ & $1.408(13)$ & $\mathrm{C}(10)-\mathrm{C}(11 \mathrm{~B})-\mathrm{C}(12 \mathrm{~B})$ & $126(2)$ \\
\hline$C(13)-S(2)$ & $1.785(10)$ & $C(7)-C(12 A)-C(11 A)$ & $120(2)$ \\
\hline$C(14)-C(15)$ & $1.372(16)$ & $\mathrm{C}(7)-\mathrm{C}(12 \mathrm{~B})-\mathrm{C}(11 \mathrm{~B})$ & $121.7(19)$ \\
\hline$C(15)-C(16)$ & $1.32(2)$ & $\mathrm{C}(7)-\mathrm{C}(12 \mathrm{~B})-\mathrm{C}(11 \mathrm{~A})$ & $89.2(15)$ \\
\hline$C(16)-C(17)$ & $1.39(3)$ & $\mathrm{C}(11 \mathrm{~B})-\mathrm{C}(12 \mathrm{~B})-\mathrm{C}(11 \mathrm{~A})$ & $44.6(14)$ \\
\hline $\mathrm{C}(17)-\mathrm{C}(18)$ & $1.413(18)$ & $\mathrm{C}(18)-\mathrm{C}(13)-\mathrm{C}(14)$ & $120.5(10)$ \\
\hline $\mathrm{Cl}(1)-\mathrm{Pt}$ & $2.318(2)$ & $\mathrm{C}(18)-\mathrm{C}(13)-\mathrm{S}(2)$ & $118.4(8)$ \\
\hline $\mathrm{Cl}(2)-\mathrm{Pt}$ & $2.311(2)$ & $\mathrm{C}(14)-\mathrm{C}(13)-\mathrm{S}(2)$ & $120.8(9)$ \\
\hline $\mathrm{Pt}-\mathrm{S}(2)$ & $2.267(2)$ & $\mathrm{C}(15)-\mathrm{C}(14)-\mathrm{C}(13)$ & $119.6(11)$ \\
\hline $\mathrm{Pt}-\mathrm{S}(1)$ & $2.281(2)$ & $C(16)-C(15)-C(14)$ & $120.6(11)$ \\
\hline \multirow[t]{2}{*}{$\operatorname{Si}(1)-\operatorname{Si}(2)$} & $2.357(4)$ & $C(15)-C(16)-C(17)$ & $121.8(12)$ \\
\hline & & $C(16)-C(17)-C(18)$ & $118.9(17)$ \\
\hline $\mathrm{S}(1)-\mathrm{C}(1)-\mathrm{Si}(1)$ & $111.8(4)$ & $C(13)-C(18)-C(17)$ & $118.7(13)$ \\
\hline $\mathrm{S}(2)-\mathrm{C}(2)-\mathrm{Si}(2)$ & $114.0(5)$ & $\mathrm{S}(2)-\mathrm{Pt}-\mathrm{S}(1)$ & $91.15(8)$ \\
\hline
\end{tabular}




$\begin{array}{lrll}\mathrm{S}(2)-\mathrm{Pt}-\mathrm{Cl}(2) & 92.51(9) & \mathrm{C}(3)-\mathrm{Si}(1)-\mathrm{C}(1) & 105.0(4) \\ \mathrm{S}(1)-\mathrm{Pt}-\mathrm{Cl}(2) & 176.32(7) & \mathrm{C}(4)-\mathrm{Si}(1)-\mathrm{C}(1) & 108.0(5) \\ \mathrm{S}(2)-\mathrm{Pt}-\mathrm{Cl}(1) & 177.14(8) & \mathrm{C}(3)-\mathrm{Si}(1)-\mathrm{Si}(2) & 108.2(4) \\ \mathrm{S}(1)-\mathrm{Pt}-\mathrm{Cl}(1) & 86.29(9) & \mathrm{C}(4)-\mathrm{Si}(1)-\mathrm{Si}(2) & 109.8(4) \\ \mathrm{Cl}(2)-\mathrm{Pt}-\mathrm{Cl}(1) & 90.04(9) & \mathrm{C}(1)-\mathrm{Si}(1)-\mathrm{Si}(2) & 115.0(3) \\ \mathrm{C}(1)-\mathrm{S}(1)-\mathrm{C}(7) & 102.9(4) & \mathrm{C}(6)-\mathrm{Si}(2)-\mathrm{C}(5) & 110.2(6) \\ \mathrm{C}(1)-\mathrm{S}(1)-\mathrm{Pt} & 110.6(3) & \mathrm{C}(6)-\mathrm{Si}(2)-\mathrm{C}(2) & 107.6(5) \\ \mathrm{C}(7)-\mathrm{S}(1)-\mathrm{Pt} & 109.4(3) & \mathrm{C}(5)-\mathrm{Si}(2)-\mathrm{C}(2) & 104.1(5) \\ \mathrm{C}(13)-\mathrm{S}(2)-\mathrm{C}(2) & 104.3(5) & \mathrm{C}(6)-\mathrm{Si}(2)-\mathrm{Si}(1) & 109.4(4) \\ \mathrm{C}(13)-\mathrm{S}(2)-\mathrm{Pt} & 108.9(4) & \mathrm{C}(5)-\mathrm{Si}(2)-\mathrm{Si}(1) & 108.3(4) \\ \mathrm{C}(2)-\mathrm{S}(2)-\mathrm{Pt} & 107.4(3) & \mathrm{C}(2)-\mathrm{Si}(2)-\mathrm{Si}(1) & 117.1(3) \\ \mathrm{C}(3)-\mathrm{Si}(1)-\mathrm{C}(4) & 110.9(5) & & \end{array}$

Symmetry transformations used to generate equivalent atoms: 
Table 4. Anisotropic displacement parameters $\left(\AA^{2} \times 10^{3}\right)$ for 2a. The anisotropic displacement factor exponent takes the form: $-2 \pi^{2}\left[h^{2} a^{* 2} U^{11}+\ldots+2 h k a^{*} b^{*} U^{12}\right]$

\begin{tabular}{|c|c|c|c|c|c|c|}
\hline & $\mathrm{U}^{11}$ & $\mathrm{U}^{22}$ & $\mathrm{U}^{33}$ & $\mathrm{U}^{23}$ & $\mathrm{U}^{13}$ & $\mathrm{U}^{12}$ \\
\hline $\mathrm{C}(1)$ & $20(4)$ & $24(5)$ & $27(4)$ & $3(4)$ & $5(3)$ & $15(4)$ \\
\hline $\mathrm{C}(2)$ & $18(4)$ & $31(5)$ & $30(4)$ & $9(4)$ & $9(3)$ & $5(4)$ \\
\hline$C(3)$ & $39(6)$ & $41(6)$ & $33(5)$ & $5(4)$ & $7(4)$ & $18(5)$ \\
\hline$C(4)$ & $32(5)$ & $36(6)$ & $38(5)$ & $-8(4)$ & $-5(4)$ & $14(5)$ \\
\hline$C(5)$ & $61(8)$ & $45(8)$ & $46(6)$ & $7(5)$ & $31(6)$ & $33(6)$ \\
\hline$C(6)$ & $61(8)$ & $30(6)$ & $41(6)$ & $3(5)$ & $-14(6)$ & $16(6)$ \\
\hline$C(7)$ & $17(4)$ & $35(5)$ & $22(4)$ & $9(4)$ & $3(3)$ & $5(4)$ \\
\hline $\mathrm{C}(8 \mathrm{~A})$ & $44(12)$ & $57(15)$ & $23(9)$ & $18(10)$ & $1(8)$ & $25(11)$ \\
\hline $\mathrm{C}(8 \mathrm{~B})$ & $27(10)$ & $51(14)$ & $31(10)$ & $22(10)$ & $5(8)$ & $4(9)$ \\
\hline$C(9 A)$ & $77(19)$ & $37(14)$ & $35(11)$ & $-2(10)$ & $13(11)$ & $24(13)$ \\
\hline $\mathrm{C}(9 \mathrm{~B})$ & $30(12)$ & $120(30)$ & $39(12)$ & $28(15)$ & $22(10)$ & $32(14)$ \\
\hline$C(10)$ & $48(7)$ & $82(11)$ & $32(6)$ & $-3(6)$ & $2(5)$ & $32(7)$ \\
\hline$C(11 A)$ & $54(15)$ & $38(13)$ & $48(13)$ & $2(11)$ & $-4(11)$ & $32(12)$ \\
\hline$C(11 B)$ & $43(13)$ & $34(12)$ & $45(12)$ & $5(10)$ & $-9(10)$ & $8(10)$ \\
\hline $\mathrm{C}(12 \mathrm{~A})$ & $54(14)$ & $45(14)$ & $35(11)$ & $7(10)$ & $-2(10)$ & $32(12)$ \\
\hline$C(12 B)$ & $33(11)$ & $55(15)$ & $31(10)$ & $12(10)$ & $5(8)$ & $2(10)$ \\
\hline$C(13)$ & $26(5)$ & $21(5)$ & $29(4)$ & $4(4)$ & $-6(4)$ & $-8(4)$ \\
\hline$C(14)$ & $26(5)$ & $27(6)$ & $50(6)$ & $12(5)$ & $1(4)$ & $-7(4)$ \\
\hline$C(15)$ & $37(6)$ & $28(6)$ & $59(7)$ & $7(5)$ & $-15(5)$ & $-15(5)$ \\
\hline$C(16)$ & $128(16)$ & $31(7)$ & $52(8)$ & $-9(6)$ & $-17(9)$ & $-31(9)$ \\
\hline$C(17)$ & $220(30)$ & $40(9)$ & $53(9)$ & $-22(7)$ & $33(13)$ & $-25(14)$ \\
\hline$C(18)$ & $123(14)$ & $24(6)$ & $49(7)$ & $-11(5)$ & $35(8)$ & $-32(8)$ \\
\hline $\mathrm{Cl}(1)$ & $9(1)$ & $36(1)$ & $55(2)$ & $14(1)$ & $3(1)$ & $2(1)$ \\
\hline $\mathrm{Cl}(2)$ & $32(1)$ & $29(1)$ & $45(1)$ & $17(1)$ & $5(1)$ & $12(1)$ \\
\hline $\mathrm{Pt}$ & $11(1)$ & $16(1)$ & $21(1)$ & $3(1)$ & $2(1)$ & $2(1)$ \\
\hline $\mathrm{S}(1)$ & $14(1)$ & $20(1)$ & $24(1)$ & $6(1)$ & $2(1)$ & $1(1)$ \\
\hline $\mathrm{S}(2)$ & $10(1)$ & $21(1)$ & $26(1)$ & $5(1)$ & $2(1)$ & $-1(1)$ \\
\hline $\operatorname{Si}(1)$ & $25(1)$ & $22(1)$ & $25(1)$ & $0(1)$ & $2(1)$ & $8(1)$ \\
\hline $\operatorname{Si}(2)$ & $33(1)$ & $26(1)$ & $23(1)$ & $2(1)$ & $7(1)$ & $13(1)$ \\
\hline
\end{tabular}


Table 5. Hydrogen coordinates ( $\left.\times 10^{4}\right)$ and isotropic displacement parameters $\left(\AA^{2} \times 10^{3}\right)$ for $2 \mathbf{a}$.

\begin{tabular}{|c|c|c|c|c|}
\hline & $\mathrm{x}$ & $\mathrm{y}$ & $\mathrm{z}$ & $\mathrm{U}(\mathrm{eq})$ \\
\hline $\mathrm{H}(1 \mathrm{~A})$ & 1633 & 2873 & 2195 & 27 \\
\hline $\mathrm{H}(1 \mathrm{~B})$ & 2872 & 4511 & 2166 & 27 \\
\hline $\mathrm{H}(2 \mathrm{~A})$ & 1543 & -842 & 3638 & 31 \\
\hline $\mathrm{H}(2 \mathrm{~B})$ & -229 & -265 & 3429 & 31 \\
\hline $\mathrm{H}(3 \mathrm{~A})$ & 1881 & 5471 & 4392 & 54 \\
\hline $\mathrm{H}(3 \mathrm{~B})$ & 499 & 4813 & 3621 & 54 \\
\hline $\mathrm{H}(3 \mathrm{C})$ & 2258 & 6170 & 3596 & 54 \\
\hline $\mathrm{H}(4 \mathrm{~A})$ & 6111 & 5682 & 3527 & 54 \\
\hline $\mathrm{H}(4 \mathrm{~B})$ & 6429 & 4071 & 3569 & 54 \\
\hline $\mathrm{H}(4 \mathrm{C})$ & 5907 & 5029 & 4342 & 54 \\
\hline $\mathrm{H}(5 \mathrm{~A})$ & -53 & 1311 & 5062 & 70 \\
\hline $\mathrm{H}(5 \mathrm{~B})$ & -828 & 2095 & 4404 & 70 \\
\hline $\mathrm{H}(5 \mathrm{C})$ & 660 & 3067 & 5088 & 70 \\
\hline $\mathrm{H}(6 \mathrm{~A})$ & 4676 & 2479 & 5082 & 66 \\
\hline $\mathrm{H}(6 \mathrm{~B})$ & 5084 & 1317 & 4372 & 66 \\
\hline $\mathrm{H}(6 \mathrm{C})$ & 3689 & 749 & 5017 & 66 \\
\hline $\mathrm{H}(8 \mathrm{~A})$ & 4980 & 466 & 724 & 46 \\
\hline $\mathrm{H}(8 \mathrm{~B})$ & 5785 & 3980 & 594 & 43 \\
\hline $\mathrm{H}(9 \mathrm{~A})$ & 4065 & -243 & -659 & 58 \\
\hline $\mathrm{H}(9 \mathrm{~B})$ & 4918 & 3462 & -799 & 69 \\
\hline $\mathrm{H}(10)$ & $2340(160)$ & $1410(140)$ & $-1330(70)$ & $30(30)$ \\
\hline $\mathrm{H}(11 \mathrm{~A})$ & 1234 & 2879 & -477 & 53 \\
\hline $\mathrm{H}(11 \mathrm{~B})$ & 672 & 202 & -398 & 49 \\
\hline $\mathrm{H}(12 \mathrm{~A})$ & 2176 & 3643 & 824 & 50 \\
\hline $\mathrm{H}(12 \mathrm{~B})$ & 1443 & 735 & 929 & 49 \\
\hline $\mathrm{H}(14)$ & -888 & -2666 & 2756 & 44 \\
\hline $\mathrm{H}(15)$ & -1885 & -5101 & 2116 & 55 \\
\hline $\mathrm{H}(16)$ & -607 & -5869 & 1009 & 98 \\
\hline $\mathrm{H}(17)$ & 1782 & -4263 & 479 & 140 \\
\hline $\mathrm{H}(18)$ & 2862 & -1762 & 1122 & 90 \\
\hline
\end{tabular}


ORTEP Plot for 2a (50\% probability ellipsoids).

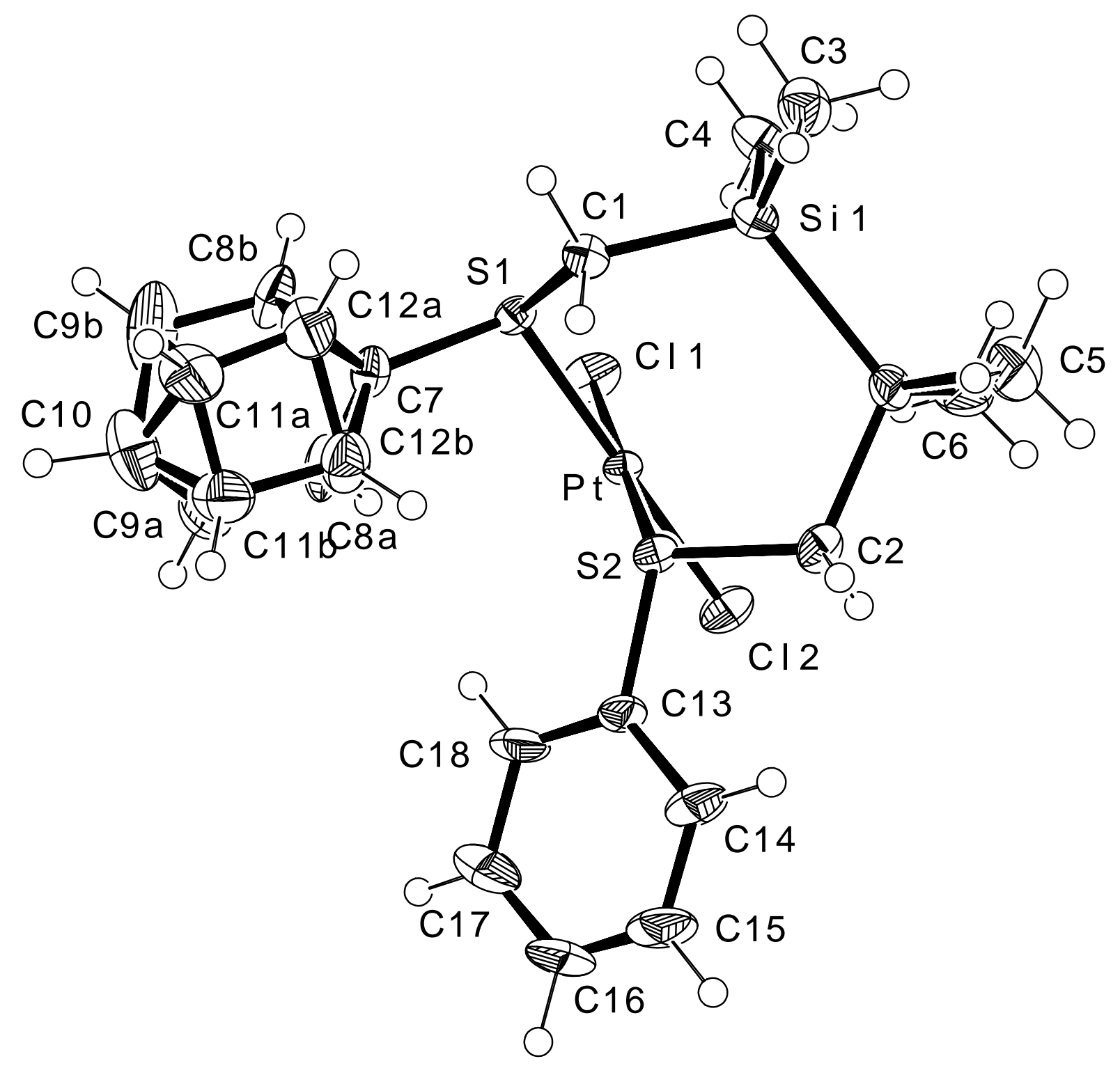


Table 6. Crystal data and structure refinement for $\mathbf{2} \mathbf{b}$.

Identification code

Empirical formula

Formula weight

Temperature

Wavelength

Crystal system

Space group

Unit cell dimensions

Volume

Z

Density (calculated)

Absorption coefficient

$\mathrm{F}(000)$

Crystal size

Theta range for data collection

Index ranges

Reflections collected

Independent reflections

Completeness to theta $=26.99^{\circ}$

Max. and min. transmission

Refinement method

Data / restraints / parameters

Goodness-of-fit on $\mathrm{F}^{2}$

Final $\mathrm{R}$ indices [I $>2 \operatorname{sigma}(\mathrm{I})]$

$\mathrm{R}$ indices (all data)

Largest diff. peak and hole 2b

C19 H28 Cl2 I2 Pt S2 Si2

896.50

173(2) K

$0.71073 \AA$

Monoclinic

$\mathrm{P} 2(1) / \mathrm{c}$

$$
\begin{array}{ll}
\mathrm{a}=12.815(4) \AA & \alpha=90^{\circ} . \\
\mathrm{b}=11.1543(14) \AA & \beta=101.93(3)^{\circ} . \\
\mathrm{c}=19.862(4) \AA & \gamma=90^{\circ} .
\end{array}
$$

$2777.6(11) \AA^{3}$

4

$2.144 \mathrm{Mg} / \mathrm{m}^{3}$

$7.710 \mathrm{~mm}^{-1}$

1680

$0.30 \times 0.20 \times 0.20 \mathrm{~mm}^{3}$

2.44 to $26.99^{\circ}$.

$-16<=\mathrm{h}<=16,-14<=\mathrm{k}<=14,-25<=\mathrm{l}<=23$

25065

$6019[\mathrm{R}($ int $)=0.0496]$

$99.2 \%$

0.3077 and 0.2057

Full-matrix least-squares on $\mathrm{F}^{2}$

$6019 / 0 / 257$

1.031

$\mathrm{R} 1=0.0421, \mathrm{wR} 2=0.1057$

$\mathrm{R} 1=0.0447, \mathrm{wR} 2=0.1080$

1.952 (near Pt and I) and -3.196 e. $\AA^{-3}$ 
Table 7. Atomic coordinates $\left(\mathrm{x} 10^{4}\right)$ and equivalent isotropic displacement parameters $\left(\AA^{2} \times 10^{3}\right)$ for $\mathbf{2 b}$. $U(e q)$ is defined as one third of the trace of the orthogonalized $U^{i j}$ tensor.

\begin{tabular}{|c|c|c|c|c|}
\hline & $\mathrm{x}$ & $\mathrm{y}$ & $\mathrm{z}$ & $\mathrm{U}(\mathrm{eq})$ \\
\hline $\mathrm{C}(1)$ & $7736(6)$ & $294(6)$ & $8939(3)$ & $37(1)$ \\
\hline$C(2)$ & $7147(5)$ & 2554(6) & $8073(4)$ & $41(2)$ \\
\hline$C(3)$ & 9099(4) & $1052(5)$ & 7977(3) & $26(1)$ \\
\hline$C(4)$ & 10573(4) & $1543(5)$ & $7133(3)$ & $22(1)$ \\
\hline$C(5)$ & $11255(4)$ & $794(5)$ & $7579(3)$ & $26(1)$ \\
\hline $\mathrm{C}(6)$ & $12237(5)$ & $521(5)$ & 7433(3) & $33(1)$ \\
\hline$C(7)$ & $12536(5)$ & $970(6)$ & $6850(4)$ & $38(1)$ \\
\hline $\mathrm{C}(8)$ & 11841(5) & $1710(6)$ & 6414(4) & $39(1)$ \\
\hline$C(9)$ & $10875(5)$ & $2009(6)$ & $6560(3)$ & $33(1)$ \\
\hline $\mathrm{C}(10)$ & $5732(6)$ & $-1174(6)$ & $7722(4)$ & $41(2)$ \\
\hline $\mathrm{C}(11)$ & $5572(5)$ & $777(7)$ & $6651(4)$ & $40(1)$ \\
\hline $\mathrm{C}(12)$ & $7249(5)$ & $-1244(5)$ & $6765(3)$ & $27(1)$ \\
\hline$C(13)$ & $8947(4)$ & $-1626(5)$ & $6068(3)$ & $22(1)$ \\
\hline$C(14)$ & $8434(4)$ & $-2684(5)$ & $5838(3)$ & $25(1)$ \\
\hline$C(15)$ & $8830(5)$ & $-3415(5)$ & 5394(3) & $30(1)$ \\
\hline$C(16)$ & $9750(5)$ & $-3092(5)$ & $5175(3)$ & $31(1)$ \\
\hline $\mathrm{C}(17)$ & $10256(5)$ & $-2028(5)$ & $5404(3)$ & $34(1)$ \\
\hline$C(18)$ & $9873(5)$ & $-1301(5)$ & $5855(3)$ & $28(1)$ \\
\hline$C(19)$ & $5030(11)$ & 7977(19) & $219(7)$ & $150(9)$ \\
\hline $\mathrm{I}(1)$ & 7839(1) & $3432(1)$ & $5895(1)$ & $31(1)$ \\
\hline $\mathrm{I}(2)$ & 7098(1) & $430(1)$ & $5104(1)$ & $30(1)$ \\
\hline $\mathrm{Pt}$ & $8215(1)$ & $1213(1)$ & $6268(1)$ & $18(1)$ \\
\hline $\mathrm{S}(1)$ & $9282(1)$ & 1938(1) & $7259(1)$ & $20(1)$ \\
\hline$S(2)$ & $8524(1)$ & $-688(1)$ & $6680(1)$ & $20(1)$ \\
\hline $\operatorname{Si}(1)$ & $7660(1)$ & $1000(1)$ & $8082(1)$ & $22(1)$ \\
\hline $\operatorname{Si}(2)$ & $6529(1)$ & $-174(1)$ & $7257(1)$ & $25(1)$ \\
\hline $\mathrm{Cl}(1)$ & $5390(2)$ & $8558(3)$ & $1069(2)$ & $92(1)$ \\
\hline $\mathrm{Cl}(2)$ & $5854(3)$ & $8360(4)$ & $-301(2)$ & 104(1) \\
\hline
\end{tabular}


Table 8. Bond lengths $[\AA]$ and angles $\left[{ }^{\circ}\right]$ for $\mathbf{2 b}$.

\begin{tabular}{|c|c|c|c|}
\hline$C(1)-\operatorname{Si}(1)$ & $1.860(6)$ & $C(5)-C(6)-C(7)$ & 121.1(6) \\
\hline $\mathrm{C}(2)-\mathrm{Si}(1)$ & $1.853(6)$ & $C(8)-C(7)-C(6)$ & $119.1(6)$ \\
\hline $\mathrm{C}(3)-\mathrm{S}(1)$ & $1.790(6)$ & $\mathrm{C}(9)-\mathrm{C}(8)-\mathrm{C}(7)$ & $120.5(6)$ \\
\hline $\mathrm{C}(3)-\mathrm{Si}(1)$ & $1.899(6)$ & $\mathrm{C}(8)-\mathrm{C}(9)-\mathrm{C}(4)$ & $120.2(6)$ \\
\hline$C(4)-C(9)$ & $1.378(8)$ & $\mathrm{S}(2)-\mathrm{C}(12)-\mathrm{Si}(2)$ & $112.4(3)$ \\
\hline$C(4)-C(5)$ & $1.388(8)$ & $C(14)-C(13)-C(18)$ & $120.1(5)$ \\
\hline $\mathrm{C}(4)-\mathrm{S}(1)$ & $1.779(5)$ & $\mathrm{C}(14)-\mathrm{C}(13)-\mathrm{S}(2)$ & $122.6(4)$ \\
\hline$C(5)-C(6)$ & $1.382(8)$ & $\mathrm{C}(18)-\mathrm{C}(13)-\mathrm{S}(2)$ & $117.2(4)$ \\
\hline$C(6)-C(7)$ & $1.388(10)$ & $C(15)-C(14)-C(13)$ & $120.4(5)$ \\
\hline$C(7)-C(8)$ & $1.381(10)$ & $C(14)-C(15)-C(16)$ & $119.9(5)$ \\
\hline$C(8)-C(9)$ & $1.370(9)$ & $C(17)-C(16)-C(15)$ & $119.4(5)$ \\
\hline$C(10)-\operatorname{Si}(2)$ & $1.878(7)$ & $C(18)-C(17)-C(16)$ & $121.0(6)$ \\
\hline $\mathrm{C}(11)-\mathrm{Si}(2)$ & $1.862(7)$ & $C(17)-C(18)-C(13)$ & $119.2(5)$ \\
\hline $\mathrm{C}(12)-\mathrm{S}(2)$ & $1.788(6)$ & $\mathrm{Cl}(2)-\mathrm{C}(19)-\mathrm{Cl}(1)$ & $114.8(7)$ \\
\hline $\mathrm{C}(12)-\mathrm{Si}(2)$ & $1.898(6)$ & $\mathrm{S}(2)-\mathrm{Pt}-\mathrm{S}(1)$ & $89.48(5)$ \\
\hline$C(13)-C(14)$ & $1.381(7)$ & $\mathrm{S}(2)-\mathrm{Pt}-\mathrm{I}(1)$ & $175.36(3)$ \\
\hline$C(13)-C(18)$ & $1.387(8)$ & S(1)-Pt-I(1) & $87.20(3)$ \\
\hline $\mathrm{C}(13)-\mathrm{S}(2)$ & $1.774(5)$ & S(2)-Pt-I(2) & 91.73(3) \\
\hline$C(14)-C(15)$ & $1.373(8)$ & S(1)-Pt-I(2) & $176.42(3)$ \\
\hline$C(15)-C(16)$ & $1.386(9)$ & I(1)-Pt-I(2) & $91.771(18)$ \\
\hline$C(16)-C(17)$ & $1.383(9)$ & $C(4)-S(1)-C(3)$ & $104.7(3)$ \\
\hline$C(17)-C(18)$ & $1.372(9)$ & $\mathrm{C}(4)-\mathrm{S}(1)-\mathrm{Pt}$ & $101.47(17)$ \\
\hline $\mathrm{C}(19)-\mathrm{Cl}(2)$ & $1.679(15)$ & $\mathrm{C}(3)-\mathrm{S}(1)-\mathrm{Pt}$ & $109.56(19)$ \\
\hline $\mathrm{C}(19)-\mathrm{Cl}(1)$ & $1.776(17)$ & $\mathrm{C}(13)-\mathrm{S}(2)-\mathrm{C}(12)$ & $106.0(2)$ \\
\hline $\mathrm{I}(1)-\mathrm{Pt}$ & $2.6004(5)$ & $\mathrm{C}(13)-\mathrm{S}(2)-\mathrm{Pt}$ & $110.95(18)$ \\
\hline $\mathrm{I}(2)-\mathrm{Pt}$ & $2.6042(8)$ & $\mathrm{C}(12)-\mathrm{S}(2)-\mathrm{Pt}$ & $105.24(19)$ \\
\hline $\mathrm{Pt}-\mathrm{S}(2)$ & $2.2773(12)$ & $\mathrm{C}(2)-\mathrm{Si}(1)-\mathrm{C}(1)$ & $110.9(3)$ \\
\hline $\mathrm{Pt}-\mathrm{S}(1)$ & $2.2990(14)$ & $\mathrm{C}(2)-\mathrm{Si}(1)-\mathrm{C}(3)$ & $108.7(3)$ \\
\hline \multirow[t]{2}{*}{$\operatorname{Si}(1)-\operatorname{Si}(2)$} & $2.348(2)$ & $C(1)-\operatorname{Si}(1)-C(3)$ & $104.2(3)$ \\
\hline & & $C(2)-\operatorname{Si}(1)-\operatorname{Si}(2)$ & $110.4(2)$ \\
\hline $\mathrm{S}(1)-\mathrm{C}(3)-\mathrm{Si}(1)$ & 113.3(3) & $\mathrm{C}(1)-\mathrm{Si}(1)-\mathrm{Si}(2)$ & $108.1(2)$ \\
\hline$C(9)-C(4)-C(5)$ & $120.5(5)$ & C(3)-Si(1)-Si(2) & $114.45(19)$ \\
\hline $\mathrm{C}(9)-\mathrm{C}(4)-\mathrm{S}(1)$ & $116.8(4)$ & $\mathrm{C}(10)-\mathrm{Si}(2)-\mathrm{C}(12)$ & $104.5(3)$ \\
\hline$C(5)-C(4)-S(1)$ & $122.7(4)$ & $C(11)-\operatorname{Si}(2)-\operatorname{Si}(1)$ & $111.2(2)$ \\
\hline $\mathrm{C}(6)-\mathrm{C}(5)-\mathrm{C}(4)$ & $118.7(5)$ & $C(12)-\operatorname{Si}(2)-\operatorname{Si}(1)$ & $114.44(19)$ \\
\hline
\end{tabular}

Symmetry transformations used to generate equivalent atoms: 
Table 9. Anisotropic displacement parameters $\left(\AA^{2} \times 10^{3}\right)$ for $\mathbf{2 b}$. The anisotropic displacement factor exponent takes the form: $-2 \pi^{2}\left[h^{2} a^{* 2} U^{11}+\ldots+2 h k a^{*} b^{*} U^{12}\right]$

\begin{tabular}{|c|c|c|c|c|c|c|}
\hline & $\mathrm{U}^{11}$ & $\mathrm{U}^{22}$ & $\mathrm{U}^{33}$ & $\mathrm{U}^{23}$ & $\mathrm{U}^{13}$ & $\mathrm{U}^{12}$ \\
\hline $\mathrm{C}(1)$ & $47(4)$ & $43(3)$ & 22(3) & $0(2)$ & $11(2)$ & $-13(3)$ \\
\hline$C(2)$ & $40(3)$ & $30(3)$ & $50(4)$ & $-8(3)$ & 2(3) & $2(2)$ \\
\hline$C(3)$ & $25(3)$ & $36(3)$ & 16(3) & $3(2)$ & $3(2)$ & $-3(2)$ \\
\hline$C(4)$ & $23(2)$ & $24(2)$ & $17(2)$ & $-3(2)$ & $4(2)$ & $-4(2)$ \\
\hline$C(5)$ & $25(2)$ & $27(3)$ & $27(3)$ & $2(2)$ & $5(2)$ & $-1(2)$ \\
\hline$C(6)$ & 28(3) & $33(3)$ & $40(3)$ & $-2(2)$ & $10(2)$ & $4(2)$ \\
\hline$C(7)$ & 23(3) & $43(3)$ & $50(4)$ & $-3(3)$ & $13(3)$ & $0(2)$ \\
\hline $\mathrm{C}(8)$ & $27(3)$ & $52(4)$ & $42(4)$ & $8(3)$ & $15(3)$ & $-8(3)$ \\
\hline $\mathrm{C}(9)$ & $34(3)$ & $39(3)$ & $28(3)$ & $9(2)$ & $9(2)$ & $-3(2)$ \\
\hline$C(10)$ & $35(3)$ & $49(4)$ & $43(4)$ & $-6(3)$ & $20(3)$ & $-14(3)$ \\
\hline $\mathrm{C}(11)$ & $27(3)$ & $50(4)$ & $40(4)$ & $-5(3)$ & $4(3)$ & 11(3) \\
\hline$C(12)$ & $24(3)$ & $26(3)$ & $32(3)$ & $-2(2)$ & $11(2)$ & $-9(2)$ \\
\hline$C(13)$ & $22(2)$ & $23(2)$ & $19(2)$ & $-1(2)$ & $4(2)$ & $-2(2)$ \\
\hline $\mathrm{C}(14)$ & $28(3)$ & $26(2)$ & $22(3)$ & $-3(2)$ & $4(2)$ & $-2(2)$ \\
\hline$C(15)$ & $46(3)$ & $22(3)$ & 21(3) & $-3(2)$ & $5(2)$ & $-2(2)$ \\
\hline$C(16)$ & $38(3)$ & $34(3)$ & 20(3) & $0(2)$ & $5(2)$ & $7(2)$ \\
\hline $\mathrm{C}(17)$ & $31(3)$ & $34(3)$ & $39(3)$ & $8(2)$ & $12(2)$ & $3(2)$ \\
\hline $\mathrm{C}(18)$ & $24(3)$ & $28(3)$ & $33(3)$ & $-2(2)$ & $7(2)$ & $0(2)$ \\
\hline$C(19)$ & $93(9)$ & $240(20)$ & $92(10)$ & $78(12)$ & $-41(8)$ & $-101(12)$ \\
\hline $\mathrm{I}(1)$ & $42(1)$ & $22(1)$ & $26(1)$ & $4(1)$ & $2(1)$ & $3(1)$ \\
\hline $\mathrm{I}(2)$ & $33(1)$ & $33(1)$ & $20(1)$ & $-3(1)$ & $-5(1)$ & $-1(1)$ \\
\hline $\mathrm{Pt}$ & $18(1)$ & $19(1)$ & $15(1)$ & $0(1)$ & $2(1)$ & $-1(1)$ \\
\hline $\mathrm{S}(1)$ & $22(1)$ & $22(1)$ & $16(1)$ & $-1(1)$ & $3(1)$ & $-4(1)$ \\
\hline $\mathrm{S}(2)$ & 21(1) & 19(1) & 19(1) & $-1(1)$ & $5(1)$ & $-1(1)$ \\
\hline $\operatorname{Si}(1)$ & $24(1)$ & $24(1)$ & 19(1) & $0(1)$ & $6(1)$ & $-3(1)$ \\
\hline $\operatorname{Si}(2)$ & $18(1)$ & $32(1)$ & $26(1)$ & $-2(1)$ & $6(1)$ & $-2(1)$ \\
\hline $\mathrm{Cl}(1)$ & $72(2)$ & $103(2)$ & $103(2)$ & $24(2)$ & $21(2)$ & $40(2)$ \\
\hline $\mathrm{Cl}(2)$ & $73(2)$ & $138(3)$ & $100(2)$ & $32(2)$ & $17(2)$ & $6(2)$ \\
\hline
\end{tabular}


Table 10. Hydrogen coordinates ( $\left.\times 10^{4}\right)$ and isotropic displacement parameters $\left(\AA^{2} \times 10^{3}\right)$ for $\mathbf{2 b}$.

\begin{tabular}{|c|c|c|c|c|}
\hline & $\mathrm{x}$ & $\mathrm{y}$ & $\mathrm{z}$ & $\mathrm{U}(\mathrm{eq})$ \\
\hline $\mathrm{H}(1 \mathrm{~A})$ & 8051 & -507 & 8941 & 56 \\
\hline $\mathrm{H}(1 \mathrm{~B})$ & 7018 & 229 & 9034 & 56 \\
\hline $\mathrm{H}(1 \mathrm{C})$ & 8181 & 789 & 9294 & 56 \\
\hline $\mathrm{H}(2 \mathrm{~A})$ & 7112 & 2907 & 7617 & 61 \\
\hline $\mathrm{H}(2 \mathrm{~B})$ & 7626 & 3032 & 8420 & 61 \\
\hline $\mathrm{H}(2 \mathrm{C})$ & 6433 & 2544 & 8177 & 61 \\
\hline $\mathrm{H}(3 \mathrm{~A})$ & 9548 & 1383 & 8402 & 31 \\
\hline $\mathrm{H}(3 \mathrm{~B})$ & 9345 & 224 & 7918 & 31 \\
\hline $\mathrm{H}(5)$ & 11051 & 475 & 7976 & 31 \\
\hline $\mathrm{H}(6)$ & 12714 & 17 & 7738 & 40 \\
\hline $\mathrm{H}(7)$ & 13210 & 770 & 6751 & 45 \\
\hline $\mathrm{H}(8)$ & 12034 & 2015 & 6010 & 47 \\
\hline $\mathrm{H}(9)$ & 10411 & 2538 & 6264 & 40 \\
\hline $\mathrm{H}(10 \mathrm{~A})$ & 5251 & -684 & 7933 & 61 \\
\hline $\mathrm{H}(10 \mathrm{~B})$ & 6216 & -1622 & 8081 & 61 \\
\hline $\mathrm{H}(10 \mathrm{C})$ & 5313 & -1736 & 7395 & 61 \\
\hline $\mathrm{H}(11 \mathrm{~A})$ & 5967 & 1312 & 6402 & 59 \\
\hline $\mathrm{H}(11 \mathrm{~B})$ & 5148 & 1256 & 6910 & 59 \\
\hline $\mathrm{H}(11 \mathrm{C})$ & 5098 & 264 & 6322 & 59 \\
\hline $\mathrm{H}(12 \mathrm{~A})$ & 7344 & -2025 & 7008 & 32 \\
\hline $\mathrm{H}(12 \mathrm{~B})$ & 6803 & -1380 & 6302 & 32 \\
\hline $\mathrm{H}(14)$ & 7804 & -2907 & 5988 & 31 \\
\hline $\mathrm{H}(15)$ & 8474 & -4141 & 5237 & 36 \\
\hline $\mathrm{H}(16)$ & 10031 & -3597 & 4871 & 37 \\
\hline $\mathrm{H}(17)$ & 10878 & -1797 & 5246 & 40 \\
\hline $\mathrm{H}(18)$ & 10237 & -583 & 6020 & 33 \\
\hline $\mathrm{H}(19 \mathrm{~A})$ & 5004 & 7092 & 246 & 180 \\
\hline $\mathrm{H}(19 \mathrm{~B})$ & 4303 & 8261 & 11 & 180 \\
\hline
\end{tabular}


ORTEP Plot for $\mathbf{2 b}$ (50\% probability ellipsoids).

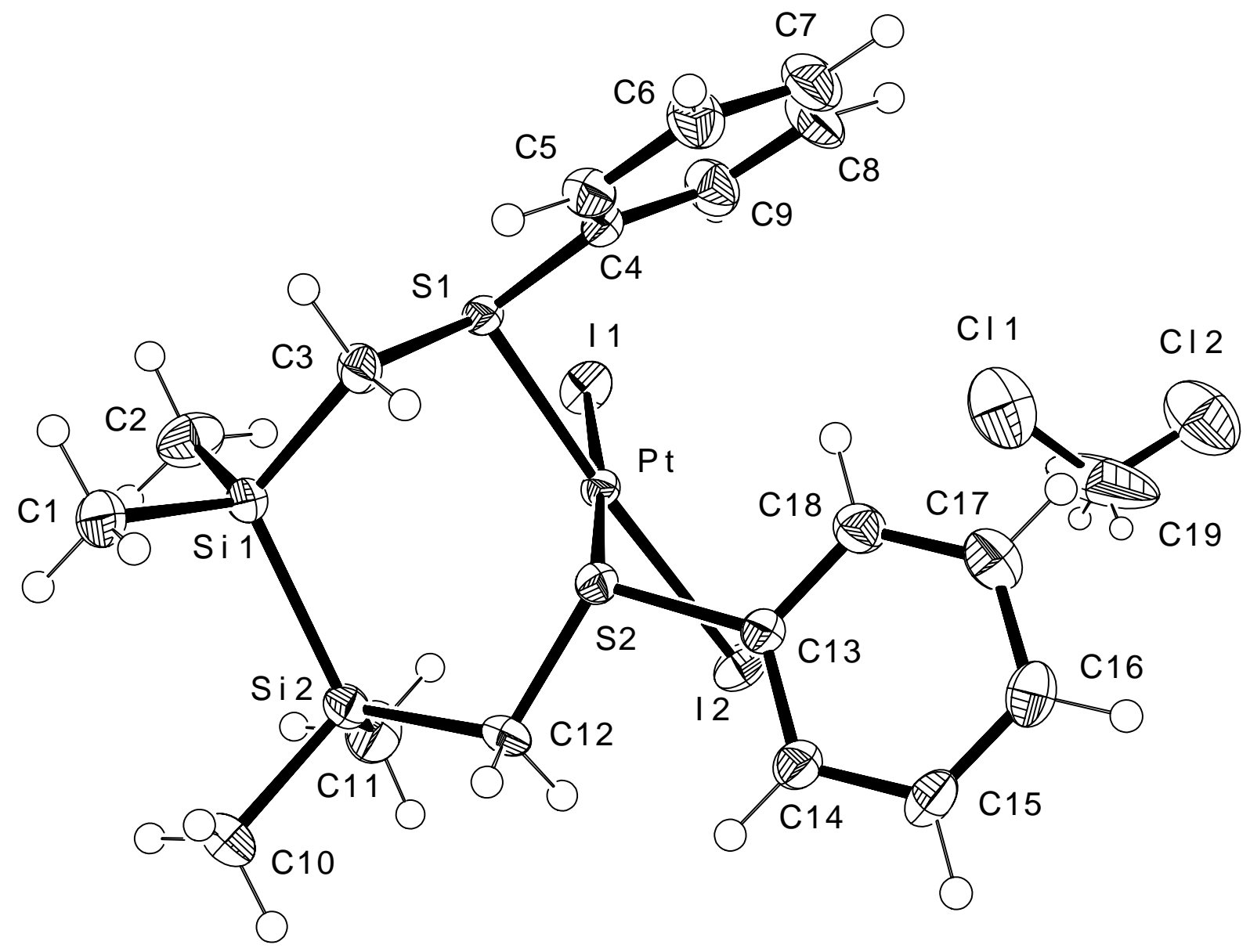


Table 11. Crystal data and structure refinement for 4.

Identification code

\section{4}

Empirical formula

C42 H54 Br2 O6 Re2 S4 Si4

Formula weight

712.83

Temperature

173(2) K

Wavelength

$0.71073 \AA$

Crystal system

Triclinic

Space group

Unit cell dimensions

P-1

$$
\begin{array}{ll}
\mathrm{a}=10.282(2) \AA & \alpha=109.11(3)^{\circ} . \\
\mathrm{b}=10.615(2) \AA & \beta=94.25(3)^{\circ} . \\
\mathrm{c}=12.989(3) \AA & \gamma=96.90(3)^{\circ} .
\end{array}
$$

Volume

$1320.0(5) \AA^{3}$

Z

Density (calculated)

1

$1.793 \mathrm{Mg} / \mathrm{m}^{3}$

Absorption coefficient

$6.385 \mathrm{~mm}^{-1}$

$\mathrm{F}(000)$

692

Crystal size

$0.30 \times 0.20 \times 0.10 \mathrm{~mm}^{3}$

Theta range for data collection

2.45 to $26.00^{\circ}$.

Index ranges

$-12<=\mathrm{h}<=11,-13<=\mathrm{k}<=13,-16<=\mathrm{l}<=16$

Reflections collected

12551

Independent reflections

$4835[\mathrm{R}($ int $)=0.0870]$

Completeness to theta $=26.00^{\circ}$

Max. and min. transmission

Refinement method

$93.3 \%$

0.5677 and 0.2504

Full-matrix least-squares on $\mathrm{F}^{2}$

Data / restraints / parameters

4835 / 0 / 275

Goodness-of-fit on $\mathrm{F}^{2}$

1.011

Final $\mathrm{R}$ indices [I $>2 \operatorname{sigma}(\mathrm{I})]$

$\mathrm{R} 1=0.0530, \mathrm{wR} 2=0.1246$

$\mathrm{R}$ indices (all data)

$\mathrm{R} 1=0.0640, \mathrm{wR} 2=0.1309$

Largest diff. peak and hole 
Table 12. Atomic coordinates ( $\left.\times 10^{4}\right)$ and equivalent isotropic displacement parameters $\left(\AA^{2} \times 10^{3}\right)$ for 4. $U(e q)$ is defined as one third of the trace of the orthogonalized $U^{i j}$ tensor.

\begin{tabular}{|c|c|c|c|c|}
\hline & $\mathrm{x}$ & $\mathrm{y}$ & $\mathrm{z}$ & $\mathrm{U}(\mathrm{eq})$ \\
\hline $\mathrm{Br}$ & $7311(1)$ & $3452(1)$ & $1520(1)$ & $41(1)$ \\
\hline$C(1)$ & $1009(11)$ & $2772(11)$ & $2608(8)$ & $53(3)$ \\
\hline$C(2)$ & $1087(11)$ & $5191(11)$ & $1818(9)$ & $54(3)$ \\
\hline$C(3)$ & $3653(9)$ & 4289(9) & $2595(7)$ & $38(2)$ \\
\hline$C(4)$ & $4177(9)$ & $6780(9)$ & $2320(6)$ & $33(2)$ \\
\hline$C(5)$ & $3877(9)$ & $7202(9)$ & $1435(7)$ & $38(2)$ \\
\hline$C(6)$ & $3400(11)$ & $8406(11)$ & $1599(8)$ & $51(2)$ \\
\hline$C(7)$ & $3205(11)$ & $9199(10)$ & $2633(8)$ & $51(2)$ \\
\hline$C(8)$ & $3494(11)$ & $8775(9)$ & $3518(8)$ & $48(2)$ \\
\hline$C(9)$ & $3971(10)$ & $7563(9)$ & $3376(6)$ & $40(2)$ \\
\hline$C(10)$ & $7095(14)$ & $9070(13)$ & $-2(10)$ & $67(3)$ \\
\hline $\mathrm{C}(11)$ & $9838(12)$ & $8379(13)$ & $550(8)$ & $62(3)$ \\
\hline$C(12)$ & $7303(10)$ & $6638(10)$ & 793(6) & $41(2)$ \\
\hline$C(13)$ & $8131(9)$ & $8716(9)$ & $2848(6)$ & $38(2)$ \\
\hline$C(14)$ & $7009(10)$ & 9308(9) & 2794(8) & $46(2)$ \\
\hline$C(15)$ & $7078(11)$ & $10707(10)$ & $3367(8)$ & $53(3)$ \\
\hline$C(16)$ & $8242(11)$ & 11461(11) & $3977(8)$ & $52(3)$ \\
\hline$C(17)$ & $9351(11)$ & $10815(10)$ & $4031(8)$ & $51(3)$ \\
\hline $\mathrm{C}(18)$ & $9295(10)$ & $9459(11)$ & $3461(7)$ & $46(2)$ \\
\hline$C(19)$ & $6393(10)$ & $4504(10)$ & $3999(6)$ & $40(2)$ \\
\hline$C(20)$ & $8778(11)$ & $5667(8)$ & $3862(7)$ & $40(2)$ \\
\hline $\mathrm{C}(21)$ & $6834(9)$ & $7122(11)$ & 4391(7) & $41(2)$ \\
\hline $\mathrm{O}(1)$ & 5991(7) & $3810(7)$ & $4484(5)$ & $48(2)$ \\
\hline $\mathrm{O}(2)$ & $9844(8)$ & $5759(7)$ & $4269(5)$ & $54(2)$ \\
\hline $\mathrm{O}(3)$ & $6782(8)$ & $8065(7)$ & $5104(5)$ & $53(2)$ \\
\hline $\mathrm{Re}$ & $7050(1)$ & $5577(1)$ & $3184(1)$ & $31(1)$ \\
\hline $\mathrm{S}(1)$ & $4836(2)$ & $5242(2)$ & $2063(2)$ & $33(1)$ \\
\hline $\mathrm{S}(2)$ & $8134(2)$ & $6956(2)$ & $2142(2)$ & $35(1)$ \\
\hline $\operatorname{Si}(1)$ & 1915(3) & $3738(3)$ & $1826(2)$ & $37(1)$ \\
\hline $\operatorname{Si}(2)$ & $8069(3)$ & $7691(3)$ & $-23(2)$ & $39(1)$ \\
\hline
\end{tabular}


Table 13. Bond lengths $[\AA]$ and angles $\left[{ }^{\circ}\right]$ for 4.

\begin{tabular}{|c|c|c|c|}
\hline Br-Re & $2.6212(13)$ & $\mathrm{C}(9)-\mathrm{C}(4)-\mathrm{S}(1)$ & $121.9(6)$ \\
\hline $\mathrm{C}(1)-\mathrm{Si}(1)$ & $1.883(9)$ & $C(6)-C(5)-C(4)$ & $120.2(8)$ \\
\hline $\mathrm{C}(2)-\mathrm{Si}(1)$ & $1.852(10)$ & $C(7)-C(6)-C(5)$ & $120.8(8)$ \\
\hline $\mathrm{C}(3)-\mathrm{S}(1)$ & $1.807(9)$ & $\mathrm{C}(6)-\mathrm{C}(7)-\mathrm{C}(8)$ & 119.2(9) \\
\hline $\mathrm{C}(3)-\mathrm{Si}(1)$ & $1.908(10)$ & $\mathrm{C}(7)-\mathrm{C}(8)-\mathrm{C}(9)$ & $121.3(8)$ \\
\hline$C(4)-C(5)$ & $1.391(11)$ & $\mathrm{C}(8)-\mathrm{C}(9)-\mathrm{C}(4)$ & $118.6(8)$ \\
\hline$C(4)-C(9)$ & $1.399(11)$ & $\mathrm{S}(2)-\mathrm{C}(12)-\mathrm{Si}(2)$ & $116.0(5)$ \\
\hline $\mathrm{C}(4)-\mathrm{S}(1)$ & $1.783(9)$ & $\mathrm{C}(18)-\mathrm{C}(13)-\mathrm{C}(14)$ & $121.2(9)$ \\
\hline$C(5)-C(6)$ & $1.382(13)$ & $\mathrm{C}(18)-\mathrm{C}(13)-\mathrm{S}(2)$ & $117.0(8)$ \\
\hline$C(6)-C(7)$ & $1.376(14)$ & $C(14)-C(13)-S(2)$ & $121.7(7)$ \\
\hline$C(7)-C(8)$ & $1.390(13)$ & $C(13)-C(14)-C(15)$ & $118.7(9)$ \\
\hline $\mathrm{C}(8)-\mathrm{C}(9)$ & $1.392(13)$ & $C(16)-C(15)-C(14)$ & $120.5(10)$ \\
\hline$C(10)-\operatorname{Si}(2)$ & $1.864(11)$ & $C(15)-C(16)-C(17)$ & $119.1(10)$ \\
\hline $\mathrm{C}(11)-\mathrm{Si}(2)$ & $1.876(12)$ & $C(18)-C(17)-C(16)$ & $120.7(10)$ \\
\hline $\mathrm{C}(12)-\mathrm{S}(2)$ & $1.799(8)$ & $\mathrm{C}(17)-\mathrm{C}(18)-\mathrm{C}(13)$ & $119.8(10)$ \\
\hline$C(12)-\operatorname{Si}(2)$ & $1.918(9)$ & $\mathrm{O}(1)-\mathrm{C}(19)-\mathrm{Re}$ & $178.3(7)$ \\
\hline $\mathrm{C}(13)-\mathrm{C}(18)$ & $1.382(13)$ & $\mathrm{O}(2)-\mathrm{C}(20)-\mathrm{Re}$ & $177.8(7)$ \\
\hline$C(13)-C(14)$ & $1.386(14)$ & $\mathrm{O}(3)-\mathrm{C}(21)-\mathrm{Re}$ & $175.9(9)$ \\
\hline$C(13)-S(2)$ & $1.792(9)$ & $C(19)-R e-C(20)$ & $88.8(4)$ \\
\hline$C(14)-C(15)$ & $1.416(13)$ & $C(19)-R e-C(21)$ & $87.5(3)$ \\
\hline$C(15)-C(16)$ & $1.383(15)$ & $\mathrm{C}(20)-\mathrm{Re}-\mathrm{C}(21)$ & $88.7(4)$ \\
\hline$C(16)-C(17)$ & $1.409(16)$ & C(19)-Re-S(2) & $174.6(3)$ \\
\hline C(17)-C(18) & $1.377(14)$ & $C(20)-R e-S(2)$ & $86.1(2)$ \\
\hline $\mathrm{C}(19)-\mathrm{O}(1)$ & $1.174(11)$ & $\mathrm{C}(21)-\mathrm{Re}-\mathrm{S}(2)$ & $93.9(3)$ \\
\hline$C(19)-R e$ & $1.894(10)$ & C(19)-Re-S(1) & $93.0(3)$ \\
\hline $\mathrm{C}(20)-\mathrm{O}(2)$ & $1.160(12)$ & C(20)-Re-S(1) & $172.6(3)$ \\
\hline$C(20)-\operatorname{Re}$ & $1.901(11)$ & $\mathrm{C}(21)-\mathrm{Re}-\mathrm{S}(1)$ & $98.5(3)$ \\
\hline $\mathrm{C}(21)-\mathrm{O}(3)$ & $1.129(11)$ & $S(2)-\operatorname{Re}-S(1)$ & $91.89(7)$ \\
\hline$C(21)-\operatorname{Re}$ & $1.911(10)$ & $\mathrm{C}(19)-\mathrm{Re}-\mathrm{Br}$ & $92.3(2)$ \\
\hline $\operatorname{Re}-\mathrm{S}(2)$ & $2.518(2)$ & $\mathrm{C}(20)-\mathrm{Re}-\mathrm{Br}$ & $92.0(3)$ \\
\hline $\operatorname{Re}-\mathrm{S}(1)$ & $2.530(2)$ & $\mathrm{C}(21)-\mathrm{Re}-\mathrm{Br}$ & $179.2(3)$ \\
\hline $\operatorname{Si}(1)-\operatorname{Si}(2) \# 1$ & $2.340(3)$ & $\mathrm{S}(2)-\mathrm{Re}-\mathrm{Br}$ & $86.38(6)$ \\
\hline \multirow[t]{2}{*}{$\operatorname{Si}(2)-\operatorname{Si}(1) \# 1$} & $2.340(3)$ & $\mathrm{S}(1)-\mathrm{Re}-\mathrm{Br}$ & $80.73(6)$ \\
\hline & & $C(4)-S(1)-C(3)$ & $101.4(4)$ \\
\hline $\mathrm{S}(1)-\mathrm{C}(3)-\mathrm{Si}(1)$ & $117.2(4)$ & $C(4)-S(1)-R e$ & $112.0(3)$ \\
\hline$C(5)-C(4)-C(9)$ & $120.0(8)$ & $\mathrm{C}(3)-\mathrm{S}(1)-\mathrm{Re}$ & $107.5(3)$ \\
\hline C(5)-C(4)-S(1) & $118.1(6)$ & $\mathrm{C}(13)-\mathrm{S}(2)-\mathrm{C}(12)$ & $104.6(4)$ \\
\hline
\end{tabular}




$\begin{array}{llll}\mathrm{C}(13)-\mathrm{S}(2)-\mathrm{Re} & 110.1(3) & \mathrm{C}(3)-\mathrm{Si}(1)-\mathrm{Si}(2) \# 1 & 111.3(3) \\ \mathrm{C}(12)-\mathrm{S}(2)-\mathrm{Re} & 114.4(3) & \mathrm{C}(10)-\mathrm{Si}(2)-\mathrm{C}(11) & 111.4(6) \\ \mathrm{C}(2)-\mathrm{Si}(1)-\mathrm{C}(1) & 109.9(5) & \mathrm{C}(10)-\mathrm{Si}(2)-\mathrm{C}(12) & 110.1(5) \\ \mathrm{C}(2)-\mathrm{Si}(1)-\mathrm{C}(3) & 112.3(5) & \mathrm{C}(11)-\mathrm{Si}(2)-\mathrm{C}(12) & 109.9(4) \\ \mathrm{C}(1)-\operatorname{Si}(1)-\mathrm{C}(3) & 104.3(4) & \mathrm{C}(10)-\mathrm{Si}(2)-\mathrm{Si}(1) \# 1 & 110.5(4) \\ \mathrm{C}(2)-\operatorname{Si}(1)-\operatorname{Si}(2) \# 1 & 109.7(4) & \mathrm{C}(11)-\mathrm{Si}(2)-\mathrm{Si}(1) \# 1 & 106.5(4) \\ \mathrm{C}(1)-\operatorname{Si}(1)-\operatorname{Si}(2) \# 1 & 109.3(4) & \mathrm{C}(12)-\operatorname{Si}(2)-\operatorname{Si}(1) \# 1 & 108.3(3)\end{array}$

Symmetry transformations used to generate equivalent atoms:

$\# 1-x+1,-y+1,-z$ 
Table 14. Anisotropic displacement parameters $\left(\AA^{2} \times 10^{3}\right)$ for 4. The anisotropic displacement factor exponent takes the form: $-2 \pi^{2}\left[h^{2} a^{* 2} U^{11}+\ldots+2 h k a^{*} b^{*} U^{12}\right]$

\begin{tabular}{|c|c|c|c|c|c|c|}
\hline & $\mathrm{U}^{11}$ & $\mathrm{U}^{22}$ & $\mathrm{U}^{33}$ & $\mathrm{U}^{23}$ & $\mathrm{U}^{13}$ & $\mathrm{U}^{12}$ \\
\hline $\mathrm{Br}$ & $51(1)$ & $34(1)$ & $38(1)$ & 11(1) & $6(1)$ & $12(1)$ \\
\hline$C(1)$ & $51(7)$ & $59(7)$ & $58(6)$ & $33(5)$ & $14(5)$ & $0(5)$ \\
\hline$C(2)$ & $44(7)$ & $49(6)$ & $78(7)$ & $31(5)$ & $13(5)$ & $12(5)$ \\
\hline$C(3)$ & $50(6)$ & $34(5)$ & $38(4)$ & $20(3)$ & $7(4)$ & $10(4)$ \\
\hline$C(4)$ & $31(5)$ & $34(5)$ & $42(4)$ & $21(3)$ & $6(3)$ & $7(3)$ \\
\hline$C(5)$ & $39(6)$ & $34(5)$ & $44(4)$ & $20(4)$ & $-1(4)$ & $3(4)$ \\
\hline$C(6)$ & $52(7)$ & $51(6)$ & $60(6)$ & $34(5)$ & $-1(4)$ & $8(5)$ \\
\hline$C(7)$ & $54(7)$ & $28(5)$ & $74(6)$ & $21(4)$ & $6(5)$ & $13(4)$ \\
\hline $\mathrm{C}(8)$ & $68(8)$ & $26(5)$ & $49(5)$ & $10(4)$ & $9(4)$ & $13(4)$ \\
\hline$C(9)$ & $51(6)$ & $37(5)$ & $33(4)$ & $15(4)$ & $6(4)$ & $3(4)$ \\
\hline$C(10)$ & $90(10)$ & $59(7)$ & $79(7)$ & $49(6)$ & $30(6)$ & $32(7)$ \\
\hline $\mathrm{C}(11)$ & $63(8)$ & $68(8)$ & $50(5)$ & $26(5)$ & $3(5)$ & $-22(6)$ \\
\hline$C(12)$ & $40(6)$ & $47(5)$ & $37(4)$ & $20(4)$ & $3(3)$ & $-1(4)$ \\
\hline$C(13)$ & $37(6)$ & $43(5)$ & $34(4)$ & $19(4)$ & $3(3)$ & $0(4)$ \\
\hline$C(14)$ & $48(7)$ & $23(5)$ & $60(5)$ & $12(4)$ & $-7(4)$ & $-3(4)$ \\
\hline$C(15)$ & $51(8)$ & $39(6)$ & $70(6)$ & $21(5)$ & $5(5)$ & $6(5)$ \\
\hline$C(16)$ & $59(8)$ & $40(6)$ & $58(6)$ & $19(4)$ & $11(5)$ & $3(5)$ \\
\hline$C(17)$ & $61(8)$ & $39(6)$ & $50(5)$ & $20(4)$ & $-2(4)$ & $-12(5)$ \\
\hline $\mathrm{C}(18)$ & $37(6)$ & $56(6)$ & $47(5)$ & $26(4)$ & $-3(4)$ & $-2(4)$ \\
\hline C(19) & $48(6)$ & $46(5)$ & $26(4)$ & $6(3)$ & $4(3)$ & $24(4)$ \\
\hline$C(20)$ & $66(7)$ & $23(4)$ & $38(4)$ & $18(3)$ & $9(4)$ & $6(4)$ \\
\hline$C(21)$ & $34(6)$ & $61(7)$ & $42(5)$ & $33(5)$ & $4(4)$ & $11(4)$ \\
\hline $\mathrm{O}(1)$ & $63(5)$ & $48(4)$ & $49(3)$ & $37(3)$ & $16(3)$ & $7(3)$ \\
\hline $\mathrm{O}(2)$ & $50(5)$ & $51(5)$ & $59(4)$ & $23(3)$ & $-14(3)$ & 1(3) \\
\hline $\mathrm{O}(3)$ & $75(6)$ & $47(4)$ & $36(3)$ & $9(3)$ & $8(3)$ & $20(4)$ \\
\hline $\mathrm{Re}$ & $40(1)$ & $26(1)$ & $31(1)$ & $14(1)$ & $4(1)$ & $6(1)$ \\
\hline$S(1)$ & $40(1)$ & $30(1)$ & $34(1)$ & $17(1)$ & $5(1)$ & $6(1)$ \\
\hline $\mathrm{S}(2)$ & $40(1)$ & $31(1)$ & $39(1)$ & $18(1)$ & $4(1)$ & $4(1)$ \\
\hline $\operatorname{Si}(1)$ & $39(2)$ & $37(1)$ & $43(1)$ & $23(1)$ & $9(1)$ & $5(1)$ \\
\hline $\operatorname{Si}(2)$ & $44(2)$ & $34(1)$ & $42(1)$ & $21(1)$ & $5(1)$ & $-1(1)$ \\
\hline
\end{tabular}


Table 15. Hydrogen coordinates $\left(\mathrm{x} 10^{4}\right)$ and isotropic displacement parameters $\left(\AA^{2} \mathrm{x} 10^{3}\right)$ for 4 .

\begin{tabular}{|c|c|c|c|c|}
\hline & $\mathrm{x}$ & $\mathrm{y}$ & $\mathrm{z}$ & $\mathrm{U}(\mathrm{eq})$ \\
\hline $\mathrm{H}(1 \mathrm{~A})$ & 991 & 3371 & 3362 & 79 \\
\hline $\mathrm{H}(1 \mathrm{~B})$ & 1462 & 2015 & 2621 & 79 \\
\hline $\mathrm{H}(1 \mathrm{C})$ & 104 & 2428 & 2250 & 79 \\
\hline $\mathrm{H}(2 \mathrm{~A})$ & 175 & 4865 & 1471 & 80 \\
\hline $\mathrm{H}(2 \mathrm{~B})$ & 1559 & 5685 & 1404 & 80 \\
\hline $\mathrm{H}(2 \mathrm{C})$ & 1090 & 5793 & 2573 & 80 \\
\hline $\mathrm{H}(3 \mathrm{~A})$ & 3585 & 4840 & 3364 & 46 \\
\hline $\mathrm{H}(3 \mathrm{~B})$ & 4011 & 3471 & 2614 & 46 \\
\hline $\mathrm{H}(5)$ & 4000 & 6660 & 716 & 46 \\
\hline $\mathrm{H}(6)$ & 3204 & 8690 & 991 & 61 \\
\hline $\mathrm{H}(7)$ & 2878 & 10027 & 2742 & 61 \\
\hline $\mathrm{H}(8)$ & 3363 & 9322 & 4233 & 57 \\
\hline $\mathrm{H}(9)$ & 4153 & 7275 & 3984 & 48 \\
\hline $\mathrm{H}(10 \mathrm{~A})$ & 7262 & 9769 & 726 & 100 \\
\hline $\mathrm{H}(10 \mathrm{~B})$ & 6152 & 8703 & -166 & 100 \\
\hline $\mathrm{H}(10 \mathrm{C})$ & 7358 & 9463 & -554 & 100 \\
\hline $\mathrm{H}(11 \mathrm{~A})$ & 10262 & 8789 & 60 & 93 \\
\hline $\mathrm{H}(11 \mathrm{~B})$ & 10298 & 7644 & 610 & 93 \\
\hline $\mathrm{H}(11 \mathrm{C})$ & 9878 & 9062 & 1278 & 93 \\
\hline $\mathrm{H}(12 \mathrm{~A})$ & 6381 & 6802 & 866 & 49 \\
\hline $\mathrm{H}(12 \mathrm{~B})$ & 7272 & 5672 & 362 & 49 \\
\hline $\mathrm{H}(14)$ & 6208 & 8785 & 2382 & 55 \\
\hline $\mathrm{H}(15)$ & 6321 & 11130 & 3332 & 64 \\
\hline $\mathrm{H}(16)$ & 8292 & 12401 & 4355 & 62 \\
\hline $\mathrm{H}(17)$ & 10147 & 11319 & 4465 & 61 \\
\hline $\mathrm{H}(18)$ & 10054 & 9037 & 3489 & 55 \\
\hline
\end{tabular}


ORTEP Plot for 4 (50\% probability ellipsoids).

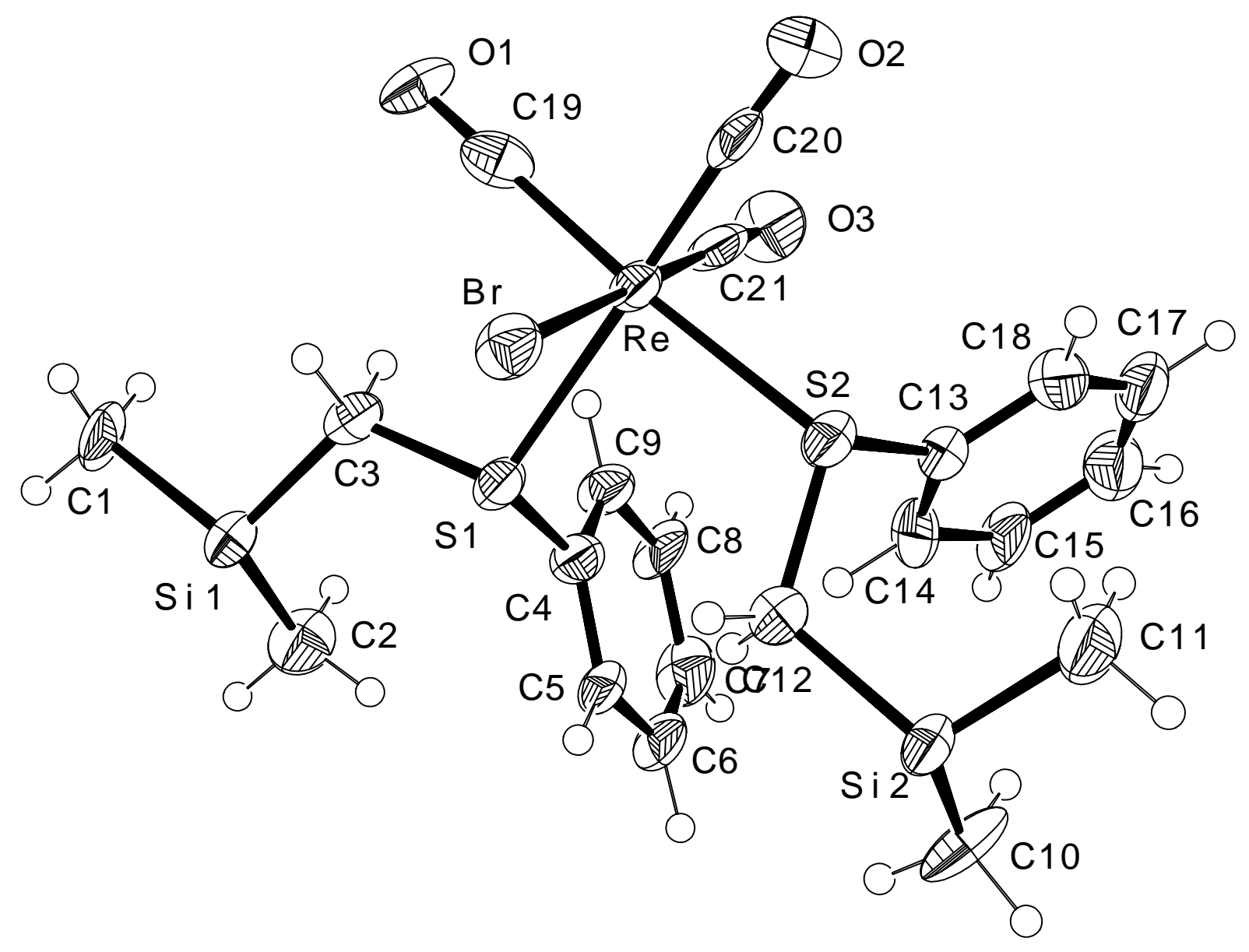


Table 16. Crystal data and structure refinement for 5.

Identification code

5

Empirical formula

C24 H26 Br2 O6 Re2 S2 Si2

Formula weight

1062.97

Temperature

173(2) K

Wavelength

$0.71073 \AA$

Crystal system

Monoclinic

Space group

Unit cell dimensions

$\mathrm{C} 2 / \mathrm{c}$

$\mathrm{a}=12.631(3) \AA$

$\alpha=90^{\circ}$.

$\mathrm{b}=18.693(4) \AA$

$\beta=100.09(3)^{\circ}$.

$\mathrm{c}=13.998(3) \AA$

$\gamma=90^{\circ}$.

Volume

$3253.8(11) \AA^{3}$

Z

Density (calculated)

$2.170 \mathrm{Mg} / \mathrm{m}^{3}$

Absorption coefficient

$10.126 \mathrm{~mm}^{-1}$

$\mathrm{F}(000)$

1992

Crystal size

$0.30 \times 0.30 \times 0.10 \mathrm{~mm}^{3}$

Theta range for data collection

2.28 to $27.00^{\circ}$.

Index ranges

$-16<=\mathrm{h}<=16,-23<=\mathrm{k}<=21,-17<=\mathrm{l}<=17$

Reflections collected

12393

Independent reflections

$3324[\mathrm{R}($ int $)=0.0705]$

Completeness to theta $=27.00^{\circ}$

$93.6 \%$

Max. and min. transmission

Refinement method

0.4308 and 0.1511

Full-matrix least-squares on $\mathrm{F}^{2}$

Data / restraints / parameters

3324 / 0 / 174

Goodness-of-fit on $\mathrm{F}^{2}$

1.058

Final $\mathrm{R}$ indices $[\mathrm{I}>2 \operatorname{sigma}(\mathrm{I})]$

$\mathrm{R} 1=0.0377, \mathrm{wR} 2=0.0909$

$\mathrm{R}$ indices (all data)

$\mathrm{R} 1=0.0412, \mathrm{wR} 2=0.0929$

Largest diff. peak and hole

1.679 and -2.286 e. $\AA^{-3}$ 
Table 17. Atomic coordinates ( $\left.\times 10^{4}\right)$ and equivalent isotropic displacement parameters $\left(\AA^{2} \times 10^{3}\right)$ for 5 . $U(e q)$ is defined as one third of the trace of the orthogonalized $U^{i j}$ tensor.

\begin{tabular}{|c|c|c|c|c|}
\hline & $\mathrm{x}$ & $\mathrm{y}$ & $\mathrm{z}$ & $\mathrm{U}(\mathrm{eq})$ \\
\hline $\mathrm{Br}$ & $5580(1)$ & $4112(1)$ & $1462(1)$ & $25(1)$ \\
\hline$C(1)$ & $2986(5)$ & $4064(3)$ & $319(5)$ & $29(1)$ \\
\hline$C(2)$ & $2121(5)$ & $4024(3)$ & $1961(5)$ & $27(1)$ \\
\hline$C(3)$ & $3597(4)$ & $3036(3)$ & $1667(4)$ & $30(1)$ \\
\hline$C(4)$ & $3318(5)$ & $7334(3)$ & $1430(5)$ & $41(2)$ \\
\hline$C(5)$ & $5596(6)$ & $7240(3)$ & $955(4)$ & $38(1)$ \\
\hline$C(6)$ & $4408(4)$ & $5880(2)$ & $1208(4)$ & $20(1)$ \\
\hline$C(7)$ & $2182(4)$ & $5670(3)$ & $1177(4)$ & $25(1)$ \\
\hline$C(8)$ & $1417(5)$ & $5835(4)$ & $1707(5)$ & $41(2)$ \\
\hline$C(9)$ & $390(6)$ & $6015(5)$ & $1262(7)$ & $57(2)$ \\
\hline$C(10)$ & $132(5)$ & $6027(4)$ & 263(6) & $50(2)$ \\
\hline $\mathrm{C}(11)$ & $914(6)$ & $5857(3)$ & $-287(6)$ & $41(2)$ \\
\hline$C(12)$ & $1938(4)$ & $5670(3)$ & 173(4) & $30(1)$ \\
\hline $\mathrm{O}(1)$ & $2632(5)$ & $4067(2)$ & $-494(4)$ & $46(1)$ \\
\hline $\mathrm{O}(2)$ & $1270(4)$ & $4001(2)$ & $2148(4)$ & $39(1)$ \\
\hline $\mathrm{O}(3)$ & $3627(4)$ & $2430(3)$ & $1660(4)$ & $50(1)$ \\
\hline $\operatorname{Re}$ & $3539(1)$ & $4065(1)$ & $1674(1)$ & $20(1)$ \\
\hline S & $3469(1)$ & $5399(1)$ & $1822(1)$ & $20(1)$ \\
\hline $\mathrm{Si}$ & $4636(1)$ & $6837(1)$ & $1665(1)$ & $23(1)$ \\
\hline
\end{tabular}


Table 18. Bond lengths $[\AA]$ and angles $\left[{ }^{\circ}\right]$ for 5 .

\begin{tabular}{|c|c|c|c|}
\hline $\mathrm{Br}-\mathrm{Re}$ & $2.6479(8)$ & $\mathrm{C}(12)-\mathrm{C}(7)-\mathrm{S}$ & $122.2(4)$ \\
\hline Br-Re\#1 & $2.6526(9)$ & $\mathrm{C}(7)-\mathrm{C}(8)-\mathrm{C}(9)$ & $121.0(7)$ \\
\hline $\mathrm{C}(1)-\mathrm{O}(1)$ & $1.147(8)$ & $C(8)-C(9)-C(10)$ & $119.9(8)$ \\
\hline$C(1)-R e$ & $1.904(6)$ & $\mathrm{C}(9)-\mathrm{C}(10)-\mathrm{C}(11)$ & $119.5(6)$ \\
\hline $\mathrm{C}(2)-\mathrm{O}(2)$ & $1.151(8)$ & $\mathrm{C}(12)-\mathrm{C}(11)-\mathrm{C}(10)$ & $119.8(7)$ \\
\hline$C(2)-\operatorname{Re}$ & $1.905(7)$ & $\mathrm{C}(11)-\mathrm{C}(12)-\mathrm{C}(7)$ & $119.7(6)$ \\
\hline $\mathrm{C}(3)-\mathrm{O}(3)$ & $1.135(8)$ & $\mathrm{C}(1)-\mathrm{Re}-\mathrm{C}(2)$ & $90.9(3)$ \\
\hline$C(3)-\operatorname{Re}$ & $1.924(6)$ & $\mathrm{C}(1)-\mathrm{Re}-\mathrm{C}(3)$ & $90.1(2)$ \\
\hline $\mathrm{C}(4)-\mathrm{Si}$ & $1.885(6)$ & $\mathrm{C}(2)-\mathrm{Re}-\mathrm{C}(3)$ & $90.0(2)$ \\
\hline $\mathrm{C}(5)-\mathrm{Si}$ & $1.857(7)$ & $C(1)-R e-S$ & $94.06(15)$ \\
\hline$C(6)-S$ & $1.820(5)$ & $C(2)-R e-S$ & $88.55(15)$ \\
\hline $\mathrm{C}(6)-\mathrm{Si}$ & $1.904(5)$ & C(3)-Re-S & $175.61(17)$ \\
\hline$C(7)-C(8)$ & $1.353(9)$ & $\mathrm{C}(1)-\mathrm{Re}-\mathrm{Br}$ & $94.73(19)$ \\
\hline$C(7)-C(12)$ & $1.385(8)$ & $\mathrm{C}(2)-\mathrm{Re}-\mathrm{Br}$ & $174.36(18)$ \\
\hline$C(7)-S$ & $1.789(5)$ & $\mathrm{C}(3)-\mathrm{Re}-\mathrm{Br}$ & $89.64(15)$ \\
\hline $\mathrm{C}(8)-\mathrm{C}(9)$ & $1.378(10)$ & S-Re-Br & $91.45(3)$ \\
\hline $\mathrm{C}(9)-\mathrm{C}(10)$ & $1.380(13)$ & $\mathrm{C}(1)-\mathrm{Re}-\mathrm{Br} \# 1$ & $176.29(19)$ \\
\hline$C(10)-C(11)$ & $1.391(12)$ & $\mathrm{C}(2)-\mathrm{Re}-\mathrm{Br} \# 1$ & $92.39(18)$ \\
\hline$C(11)-C(12)$ & $1.385(8)$ & $\mathrm{C}(3)-\mathrm{Re}-\mathrm{Br} \# 1$ & $91.65(16)$ \\
\hline Re-S & $2.5056(13)$ & S-Re-Br\#1 & $84.29(3)$ \\
\hline Re-Br\#1 & $2.6526(9)$ & Br-Re-Br\#1 & $82.01(3)$ \\
\hline \multirow[t]{2}{*}{$\mathrm{Si}-\mathrm{Si} \# 1$} & $2.357(3)$ & $C(7)-S-C(6)$ & $103.4(2)$ \\
\hline & & $\mathrm{C}(7)-\mathrm{S}-\mathrm{Re}$ & $106.41(17)$ \\
\hline Re-Br-Re\#1 & $97.87(3)$ & $C(6)-S-R e$ & $114.68(16)$ \\
\hline $\mathrm{O}(1)-\mathrm{C}(1)-\mathrm{Re}$ & $178.6(6)$ & $\mathrm{C}(5)-\mathrm{Si}-\mathrm{C}(4)$ & $110.2(3)$ \\
\hline $\mathrm{O}(2)-\mathrm{C}(2)-\mathrm{Re}$ & $179.0(6)$ & $\mathrm{C}(5)-\mathrm{Si}-\mathrm{C}(6)$ & $106.0(3)$ \\
\hline $\mathrm{O}(3)-\mathrm{C}(3)-\mathrm{Re}$ & $179.7(5)$ & $\mathrm{C}(4)-\mathrm{Si}-\mathrm{C}(6)$ & 109.1(3) \\
\hline $\mathrm{S}-\mathrm{C}(6)-\mathrm{Si}$ & $112.2(3)$ & $\mathrm{C}(5)-\mathrm{Si}-\mathrm{Si} \# 1$ & $110.7(2)$ \\
\hline$C(8)-C(7)-C(12)$ & $120.2(5)$ & C(4)-Si-Si\#1 & $110.6(2)$ \\
\hline$C(8)-C(7)-S$ & $117.5(4)$ & C(6)-Si-Si\#1 & $110.06(16)$ \\
\hline
\end{tabular}

Symmetry transformations used to generate equivalent atoms:

$\# 1-\mathrm{x}+1, \mathrm{y},-\mathrm{z}+1 / 2$ 
Table 19. Anisotropic displacement parameters $\left(\AA^{2} \times 10^{3}\right)$ for 5. The anisotropic displacement factor exponent takes the form: $-2 \pi^{2}\left[h^{2} a^{* 2} U^{11}+\ldots+2 h k a^{*} b^{*} U^{12}\right]$

\begin{tabular}{|c|c|c|c|c|c|c|}
\hline & $\mathrm{U}^{11}$ & $\mathrm{U}^{22}$ & $\mathrm{U}^{33}$ & $\mathrm{U}^{23}$ & $\mathrm{U}^{13}$ & $\mathrm{U}^{12}$ \\
\hline $\mathrm{Br}$ & $20(1)$ & $31(1)$ & $24(1)$ & $-3(1)$ & $7(1)$ & $-1(1)$ \\
\hline $\mathrm{C}(1)$ & $23(3)$ & $29(3)$ & $35(3)$ & $-4(2)$ & $8(3)$ & $2(2)$ \\
\hline$C(2)$ & $23(3)$ & $26(3)$ & $32(3)$ & $1(2)$ & $4(3)$ & $-2(2)$ \\
\hline$C(3)$ & $23(3)$ & $25(3)$ & $44(3)$ & $-2(2)$ & $8(3)$ & $-1(2)$ \\
\hline$C(4)$ & $44(3)$ & $26(3)$ & $50(3)$ & $0(3)$ & $-4(3)$ & $8(2)$ \\
\hline$C(5)$ & $54(4)$ & $28(3)$ & $38(3)$ & $-1(2)$ & $20(3)$ & $-7(3)$ \\
\hline$C(6)$ & $19(2)$ & $18(2)$ & $25(2)$ & $0(2)$ & $5(2)$ & $-2(2)$ \\
\hline$C(7)$ & $19(2)$ & $20(2)$ & $38(3)$ & $5(2)$ & $8(2)$ & $3(2)$ \\
\hline$C(8)$ & $31(3)$ & $52(4)$ & $42(3)$ & $12(3)$ & $12(3)$ & $16(3)$ \\
\hline$C(9)$ & $32(4)$ & $78(6)$ & $68(5)$ & $20(4)$ & $27(4)$ & $20(3)$ \\
\hline$C(10)$ & $22(3)$ & $59(4)$ & $67(5)$ & $32(4)$ & $2(3)$ & $10(3)$ \\
\hline $\mathrm{C}(11)$ & $32(3)$ & $45(4)$ & $44(4)$ & $18(3)$ & $0(3)$ & $-4(2)$ \\
\hline$C(12)$ & $25(2)$ & $31(3)$ & $33(3)$ & $3(2)$ & $3(2)$ & $-1(2)$ \\
\hline $\mathrm{O}(1)$ & $51(3)$ & $49(3)$ & $32(2)$ & $-8(2)$ & $-8(2)$ & $12(2)$ \\
\hline $\mathrm{O}(2)$ & $20(2)$ & $44(3)$ & $55(3)$ & $5(2)$ & $12(2)$ & $-2(2)$ \\
\hline $\mathrm{O}(3)$ & $48(3)$ & $22(2)$ & $82(4)$ & $1(2)$ & $18(3)$ & $1(2)$ \\
\hline $\mathrm{Re}$ & $15(1)$ & $18(1)$ & $25(1)$ & $-1(1)$ & $4(1)$ & $-1(1)$ \\
\hline S & $18(1)$ & $19(1)$ & $23(1)$ & $1(1)$ & $4(1)$ & $1(1)$ \\
\hline $\mathrm{Si}$ & $27(1)$ & $16(1)$ & $25(1)$ & $2(1)$ & $4(1)$ & $0(1)$ \\
\hline
\end{tabular}


Table 20. Hydrogen coordinates $\left(\mathrm{x} 10^{4}\right)$ and isotropic displacement parameters $\left(\AA^{2} \times 10^{3}\right)$ for 5 .

\begin{tabular}{lcccc}
\hline & $x$ & $y$ & $\mathrm{U}(\mathrm{eq})$ \\
& & & \\
& & & \\
$\mathrm{H}(4 \mathrm{~A})$ & 3446 & 7843 & 1576 & 62 \\
$\mathrm{H}(4 \mathrm{~B})$ & 2837 & 7139 & 1844 & 62 \\
$\mathrm{H}(4 \mathrm{C})$ & 2984 & 7279 & 747 & 62 \\
$\mathrm{H}(5 \mathrm{~A})$ & 5274 & 7244 & 265 & 58 \\
$\mathrm{H}(5 \mathrm{~B})$ & 6260 & 6958 & 1048 & 58 \\
$\mathrm{H}(5 \mathrm{C})$ & 5761 & 7732 & 1177 & 58 \\
$\mathrm{H}(6 \mathrm{~A})$ & 4126 & 5888 & 502 & 25 \\
$\mathrm{H}(6 \mathrm{~B})$ & 5105 & 5624 & 1310 & 25 \\
$\mathrm{H}(8)$ & 1590 & 5826 & 2395 & 49 \\
$\mathrm{H}(9)$ & -139 & 6131 & 1643 & 68 \\
$\mathrm{H}(10)$ & -574 & 6151 & -48 & 60 \\
$\mathrm{H}(11)$ & 747 & 5870 & -976 & 49 \\
$\mathrm{H}(12)$ & 2471 & 5542 & -198 & 36 \\
\hline & & & \\
\hline
\end{tabular}


ORTEP Plot for 5 (50\% probability ellipsoids).

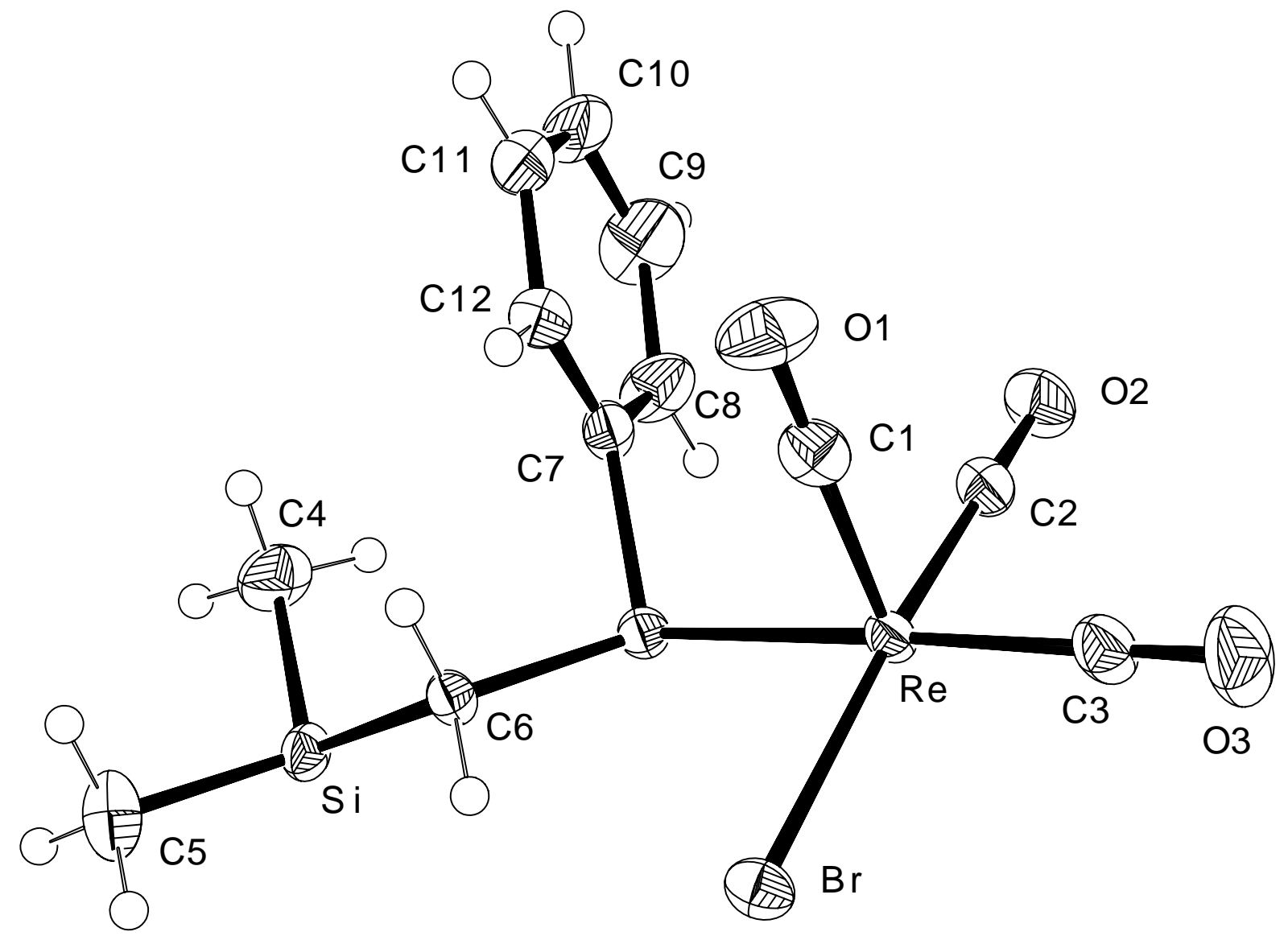


Table 21. Crystal data and structure refinement for 6 .

Identification code

Empirical formula

Formula weight

Temperature

Wavelength

Crystal system

Space group

Unit cell dimensions

Volume

Z

Density (calculated)

Absorption coefficient

$\mathrm{F}(000)$

Crystal size

Theta range for data collection

Index ranges

Reflections collected

Independent reflections

Completeness to theta $=25.00^{\circ}$

Max. and min. transmission

Refinement method

Data / restraints / parameters

Goodness-of-fit on $\mathrm{F}^{2}$

Final $\mathrm{R}$ indices [I $>2 \operatorname{sigma}(\mathrm{I})]$

$\mathrm{R}$ indices (all data)

Largest diff. peak and hole

\section{6}

C25 H28 Cl6 O6 Ru2 S2 Si2

959.61

193(2) K

$0.71073 \AA$

Triclinic

P-1

$\mathrm{a}=8.1419(8) \AA$

$\alpha=101.2800(10)^{\circ}$.

$\mathrm{b}=8.9171(9) \AA$

$\beta=90.773(2)^{\circ}$.

$\mathrm{c}=13.2822(13) \AA$

$\gamma=100.8300(10)^{\circ}$.

1

$1.718 \mathrm{Mg} / \mathrm{m}^{3}$

$1.459 \mathrm{~mm}^{-1}$

476

$0.30 \times 0.30 \times 0.20 \mathrm{~mm}^{3}$

2.55 to $25.00^{\circ}$.

$-9<=\mathrm{h}<=9,-10<=\mathrm{k}<=10,-15<=\mathrm{l}<=15$

17764

$3248[\mathrm{R}($ int $)=0.0189]$

$99.4 \%$

0.7591 and 0.6687

Full-matrix least-squares on $\mathrm{F}^{2}$

3248 / 0 / 201

1.055

$\mathrm{R} 1=0.0221, \mathrm{wR} 2=0.0574$

$\mathrm{R} 1=0.0226, \mathrm{wR} 2=0.0577$

0.968 and -0.802 e. $\AA^{-3}$ 
Table 22. Atomic coordinates ( $\left.\times 10^{4}\right)$ and equivalent isotropic displacement parameters $\left(\AA^{2} \times 10^{3}\right)$ for $6 . U(e q)$ is defined as one third of the trace of the orthogonalized $U^{i j}$ tensor.

\begin{tabular}{|c|c|c|c|c|}
\hline & $\mathrm{x}$ & $\mathrm{y}$ & $\mathrm{z}$ & $\mathrm{U}(\mathrm{eq})$ \\
\hline $\mathrm{C}(1)$ & 2475(4) & 5831(4) & 5949(2) & $45(1)$ \\
\hline $\mathrm{C}(2)$ & $5264(4)$ & 7878(3) & $5037(2)$ & $45(1)$ \\
\hline $\mathrm{C}(3)$ & 2732(3) & $5117(3)$ & $3705(2)$ & $31(1)$ \\
\hline $\mathrm{C}(4)$ & 4771(3) & $6678(3)$ & $2400(2)$ & $28(1)$ \\
\hline$C(5)$ & 3819(3) & 7815(3) & $2409(2)$ & $34(1)$ \\
\hline $\mathrm{C}(6)$ & 4599(4) & 9293(3) & 2314(2) & 41(1) \\
\hline$C(7)$ & $6310(4)$ & $9624(3)$ & $2200(2)$ & $46(1)$ \\
\hline $\mathrm{C}(8)$ & 7236(3) & $8470(3)$ & $2177(2)$ & $44(1)$ \\
\hline $\mathrm{C}(9)$ & 6482(3) & 7001(3) & $2280(2)$ & $34(1)$ \\
\hline$C(10)$ & 2791(3) & 4934(3) & $313(2)$ & $29(1)$ \\
\hline $\mathrm{C}(11)$ & $3345(3)$ & 2105(3) & $664(2)$ & $35(1)$ \\
\hline $\mathrm{C}(12)$ & $100(3)$ & 2361(3) & $105(2)$ & $37(1)$ \\
\hline $\mathrm{C}(13)$ & $9661(11)$ & $10439(12)$ & $4575(6)$ & $92(3)$ \\
\hline $\mathrm{Cl}(1)$ & 771(1) & 1689(1) & 2201(1) & $44(1)$ \\
\hline $\mathrm{Cl}(2)$ & $-49(1)$ & $5148(1)$ & 1754(1) & $37(1)$ \\
\hline $\mathrm{Cl}(3)$ & $10325(2)$ & $8578(2)$ & $4358(1)$ & $116(1)$ \\
\hline $\mathrm{O}(1)$ & $3400(2)$ & $5795(2)$ & $-144(1)$ & $41(1)$ \\
\hline $\mathrm{O}(2)$ & 4205(3) & $1280(2)$ & $412(2)$ & $52(1)$ \\
\hline $\mathrm{O}(3)$ & $-962(3)$ & 1694(2) & $-429(2)$ & $55(1)$ \\
\hline $\mathrm{Ru}$ & 1862(1) & $3484(1)$ & 1129(1) & $26(1)$ \\
\hline S & 3872(1) & 4756(1) & $2555(1)$ & $27(1)$ \\
\hline $\mathrm{Si}$ & 4074(1) & $5852(1)$ & 4950(1) & $28(1)$ \\
\hline
\end{tabular}


Table 23. Bond lengths $[\AA]$ and angles $\left[{ }^{\circ}\right]$ for 6.

\begin{tabular}{|c|c|c|c|}
\hline $\mathrm{C}(1)-\mathrm{Si}$ & $1.871(3)$ & $\mathrm{C}(9)-\mathrm{C}(8)-\mathrm{C}(7)$ & $120.7(2)$ \\
\hline $\mathrm{C}(2)-\mathrm{Si}$ & $1.864(3)$ & $C(8)-C(9)-C(4)$ & $119.3(2)$ \\
\hline$C(3)-S$ & $1.805(2)$ & $\mathrm{O}(1)-\mathrm{C}(10)-\mathrm{Ru}$ & 177.1(2) \\
\hline $\mathrm{C}(3)-\mathrm{Si}$ & $1.902(2)$ & $\mathrm{O}(2)-\mathrm{C}(11)-\mathrm{Ru}$ & 178.2(2) \\
\hline$C(4)-C(5)$ & $1.386(3)$ & $\mathrm{O}(3)-\mathrm{C}(12)-\mathrm{Ru}$ & $175.5(3)$ \\
\hline$C(4)-C(9)$ & $1.387(3)$ & $\mathrm{Cl}(3) \# 1-\mathrm{C}(13)-\mathrm{Cl}(3)$ & $121.8(4)$ \\
\hline$C(4)-S$ & $1.785(2)$ & $\mathrm{C}(13) \# 1-\mathrm{Cl}(3)-\mathrm{C}(13)$ & $58.2(4)$ \\
\hline$C(5)-C(6)$ & $1.382(4)$ & $\mathrm{C}(10)-\mathrm{Ru}-\mathrm{C}(11)$ & $93.23(10)$ \\
\hline$C(6)-C(7)$ & $1.385(4)$ & $\mathrm{C}(10)-\mathrm{Ru}-\mathrm{C}(12)$ & $94.52(10)$ \\
\hline$C(7)-C(8)$ & $1.381(4)$ & $\mathrm{C}(11)-\mathrm{Ru}-\mathrm{C}(12)$ & $93.14(11)$ \\
\hline $\mathrm{C}(8)-\mathrm{C}(9)$ & $1.372(4)$ & $\mathrm{C}(10)-\mathrm{Ru}-\mathrm{Cl}(2)$ & $88.97(7)$ \\
\hline $\mathrm{C}(10)-\mathrm{O}(1)$ & $1.119(3)$ & $\mathrm{C}(11)-\mathrm{Ru}-\mathrm{Cl}(2)$ & $177.80(7)$ \\
\hline$C(10)-R u$ & $1.903(3)$ & $\mathrm{C}(12)-\mathrm{Ru}-\mathrm{Cl}(2)$ & $86.69(8)$ \\
\hline $\mathrm{C}(11)-\mathrm{O}(2)$ & $1.117(3)$ & $\mathrm{C}(10)-\mathrm{Ru}-\mathrm{Cl}(1)$ & $178.00(7)$ \\
\hline $\mathrm{C}(11)-\mathrm{Ru}$ & $1.906(3)$ & $\mathrm{C}(11)-\mathrm{Ru}-\mathrm{Cl}(1)$ & $86.35(8)$ \\
\hline $\mathrm{C}(12)-\mathrm{O}(3)$ & $1.109(3)$ & $\mathrm{C}(12)-\mathrm{Ru}-\mathrm{Cl}(1)$ & $87.46(8)$ \\
\hline $\mathrm{C}(12)-\mathrm{Ru}$ & $1.945(3)$ & $\mathrm{Cl}(2)-\mathrm{Ru}-\mathrm{Cl}(1)$ & $91.45(3)$ \\
\hline $\mathrm{C}(13)-\mathrm{Cl}(3) \# 1$ & $1.513(10)$ & C(10)-Ru-S & $91.71(7)$ \\
\hline $\mathrm{C}(13)-\mathrm{Cl}(3)$ & $1.813(11)$ & $\mathrm{C}(11)-\mathrm{Ru}-\mathrm{S}$ & $88.67(8)$ \\
\hline $\mathrm{Cl}(1)-\mathrm{Ru}$ & $2.4018(7)$ & C(12)-Ru-S & $173.41(8)$ \\
\hline $\mathrm{Cl}(2)-\mathrm{Ru}$ & $2.3938(6)$ & $\mathrm{Cl}(2)-\mathrm{Ru}-\mathrm{S}$ & $91.26(2)$ \\
\hline $\mathrm{Cl}(3)-\mathrm{C}(13) \# 1$ & $1.513(10)$ & $\mathrm{Cl}(1)-\mathrm{Ru}-\mathrm{S}$ & $86.33(2)$ \\
\hline Ru-S & $2.4349(6)$ & $C(4)-S-C(3)$ & $102.64(11)$ \\
\hline \multirow[t]{2}{*}{$\mathrm{Si}-\mathrm{Si} \# 2$} & $2.3532(13)$ & $\mathrm{C}(4)-\mathrm{S}-\mathrm{Ru}$ & $109.73(7)$ \\
\hline & & $\mathrm{C}(3)-\mathrm{S}-\mathrm{Ru}$ & $108.16(8)$ \\
\hline $\mathrm{S}-\mathrm{C}(3)-\mathrm{Si}$ & $115.36(12)$ & $\mathrm{C}(2)-\mathrm{Si}-\mathrm{C}(1)$ & $109.79(14)$ \\
\hline $\mathrm{C}(5)-\mathrm{C}(4)-\mathrm{C}(9)$ & $120.8(2)$ & $\mathrm{C}(2)-\mathrm{Si}-\mathrm{C}(3)$ & $111.89(12)$ \\
\hline$C(5)-C(4)-S$ & $122.04(18)$ & $\mathrm{C}(1)-\mathrm{Si}-\mathrm{C}(3)$ & $102.45(12)$ \\
\hline$C(9)-C(4)-S$ & $117.16(18)$ & $\mathrm{C}(2)-\mathrm{Si}-\mathrm{Si} \# 2$ & $110.30(11)$ \\
\hline$C(6)-C(5)-C(4)$ & $119.2(2)$ & C(1)-Si-Si\#2 & $113.51(10)$ \\
\hline$C(5)-C(6)-C(7)$ & $120.2(3)$ & C(3)-Si-Si\#2 & $108.70(8)$ \\
\hline $\mathrm{C}(8)-\mathrm{C}(7)-\mathrm{C}(6)$ & $119.8(3)$ & & \\
\hline
\end{tabular}

Symmetry transformations used to generate equivalent atoms:

$\# 1-x+2,-y+2,-z+1 \quad \# 2-x+1,-y+1,-z+1$ 
Table 24. Anisotropic displacement parameters $\left(\AA^{2} \times 10^{3}\right)$ for 6 . The anisotropic displacement factor exponent takes the form: $-2 \pi^{2}\left[h^{2} a^{* 2} U^{11}+\ldots+2 h k a^{*} b^{*} U^{12}\right]$

\begin{tabular}{|c|c|c|c|c|c|c|}
\hline & $\mathrm{U}^{11}$ & $\mathrm{U}^{22}$ & $\mathrm{U}^{33}$ & $\mathrm{U}^{23}$ & $\mathrm{U}^{13}$ & $\mathrm{U}^{12}$ \\
\hline $\mathrm{C}(1)$ & $46(2)$ & $64(2)$ & $34(1)$ & $14(1)$ & $11(1)$ & $25(1)$ \\
\hline $\mathrm{C}(2)$ & $59(2)$ & $32(1)$ & $41(2)$ & $3(1)$ & $-5(1)$ & $3(1)$ \\
\hline$C(3)$ & $29(1)$ & $38(1)$ & $26(1)$ & $8(1)$ & $4(1)$ & $6(1)$ \\
\hline$C(4)$ & $28(1)$ & $32(1)$ & $21(1)$ & $4(1)$ & $-1(1)$ & $3(1)$ \\
\hline$C(5)$ & $31(1)$ & $37(1)$ & $34(1)$ & $8(1)$ & $0(1)$ & $7(1)$ \\
\hline$C(6)$ & $53(2)$ & $35(1)$ & $36(1)$ & $8(1)$ & $0(1)$ & $11(1)$ \\
\hline$C(7)$ & $55(2)$ & $36(1)$ & $40(2)$ & $9(1)$ & $1(1)$ & $-5(1)$ \\
\hline $\mathrm{C}(8)$ & $33(1)$ & $53(2)$ & $39(1)$ & $9(1)$ & $5(1)$ & $-6(1)$ \\
\hline $\mathrm{C}(9)$ & $29(1)$ & $42(1)$ & $29(1)$ & $3(1)$ & $1(1)$ & $6(1)$ \\
\hline$C(10)$ & $25(1)$ & $33(1)$ & $27(1)$ & $0(1)$ & $-2(1)$ & $8(1)$ \\
\hline $\mathrm{C}(11)$ & $41(1)$ & $31(1)$ & $33(1)$ & $8(1)$ & $4(1)$ & $7(1)$ \\
\hline$C(12)$ & $36(1)$ & $34(1)$ & $39(1)$ & $2(1)$ & $1(1)$ & $7(1)$ \\
\hline$C(13)$ & $73(5)$ & $142(8)$ & $72(5)$ & $74(6)$ & $-11(4)$ & $-7(5)$ \\
\hline $\mathrm{Cl}(1)$ & $49(1)$ & $35(1)$ & $45(1)$ & $12(1)$ & $8(1)$ & $-4(1)$ \\
\hline $\mathrm{Cl}(2)$ & $27(1)$ & $48(1)$ & $37(1)$ & $2(1)$ & $0(1)$ & $15(1)$ \\
\hline $\mathrm{Cl}(3)$ & 119(1) & 101(1) & 131(1) & $-8(1)$ & $-3(1)$ & $59(1)$ \\
\hline $\mathrm{O}(1)$ & $40(1)$ & $46(1)$ & $38(1)$ & $16(1)$ & $0(1)$ & $4(1)$ \\
\hline $\mathrm{O}(2)$ & $62(1)$ & $46(1)$ & $57(1)$ & $10(1)$ & $16(1)$ & $29(1)$ \\
\hline $\mathrm{O}(3)$ & $49(1)$ & $50(1)$ & $57(1)$ & $-5(1)$ & $-17(1)$ & $-1(1)$ \\
\hline $\mathrm{Ru}$ & $24(1)$ & $27(1)$ & $26(1)$ & $3(1)$ & $1(1)$ & $5(1)$ \\
\hline $\mathrm{S}$ & $24(1)$ & $30(1)$ & $26(1)$ & $6(1)$ & $0(1)$ & $7(1)$ \\
\hline $\mathrm{Si}$ & $33(1)$ & $30(1)$ & $24(1)$ & $5(1)$ & $2(1)$ & $9(1)$ \\
\hline
\end{tabular}


Table 25. Hydrogen coordinates ( $\left.\mathrm{x} 10^{4}\right)$ and isotropic displacement parameters $\left(\AA^{2} \times 10^{3}\right)$ for 6 .

\begin{tabular}{|c|c|c|c|c|}
\hline & $\mathrm{x}$ & $\mathrm{y}$ & $\mathrm{z}$ & $\mathrm{U}(\mathrm{eq})$ \\
\hline $\mathrm{H}(1 \mathrm{~A})$ & 3034 & 6235 & 6632 & 68 \\
\hline $\mathrm{H}(1 \mathrm{~B})$ & 1870 & 4760 & 5910 & 68 \\
\hline $\mathrm{H}(1 \mathrm{C})$ & 1683 & 6487 & 5827 & 68 \\
\hline $\mathrm{H}(2 \mathrm{~A})$ & 4491 & 8554 & 4919 & 68 \\
\hline $\mathrm{H}(2 \mathrm{~B})$ & 6105 & 7882 & 4516 & 68 \\
\hline $\mathrm{H}(2 \mathrm{C})$ & 5822 & 8265 & 5722 & 68 \\
\hline $\mathrm{H}(3 \mathrm{~A})$ & 1963 & 4137 & 3759 & 37 \\
\hline $\mathrm{H}(3 \mathrm{~B})$ & 2036 & 5892 & 3632 & 37 \\
\hline $\mathrm{H}(5)$ & 2644 & 7581 & 2479 & 41 \\
\hline $\mathrm{H}(6)$ & 3961 & 10085 & 2327 & 49 \\
\hline $\mathrm{H}(7)$ & 6845 & 10642 & 2137 & 55 \\
\hline $\mathrm{H}(8)$ & 8406 & 8696 & 2089 & 52 \\
\hline $\mathrm{H}(9)$ & 7126 & 6214 & 2270 & 41 \\
\hline $\mathrm{H}(13 \mathrm{~A})$ & 10364 & 11095 & 4161 & 111 \\
\hline $\mathrm{H}(13 \mathrm{~B})$ & 8500 & 10242 & 4277 & 111 \\
\hline
\end{tabular}


ORTEP Plot for 6 (50\% probability ellipsoids).

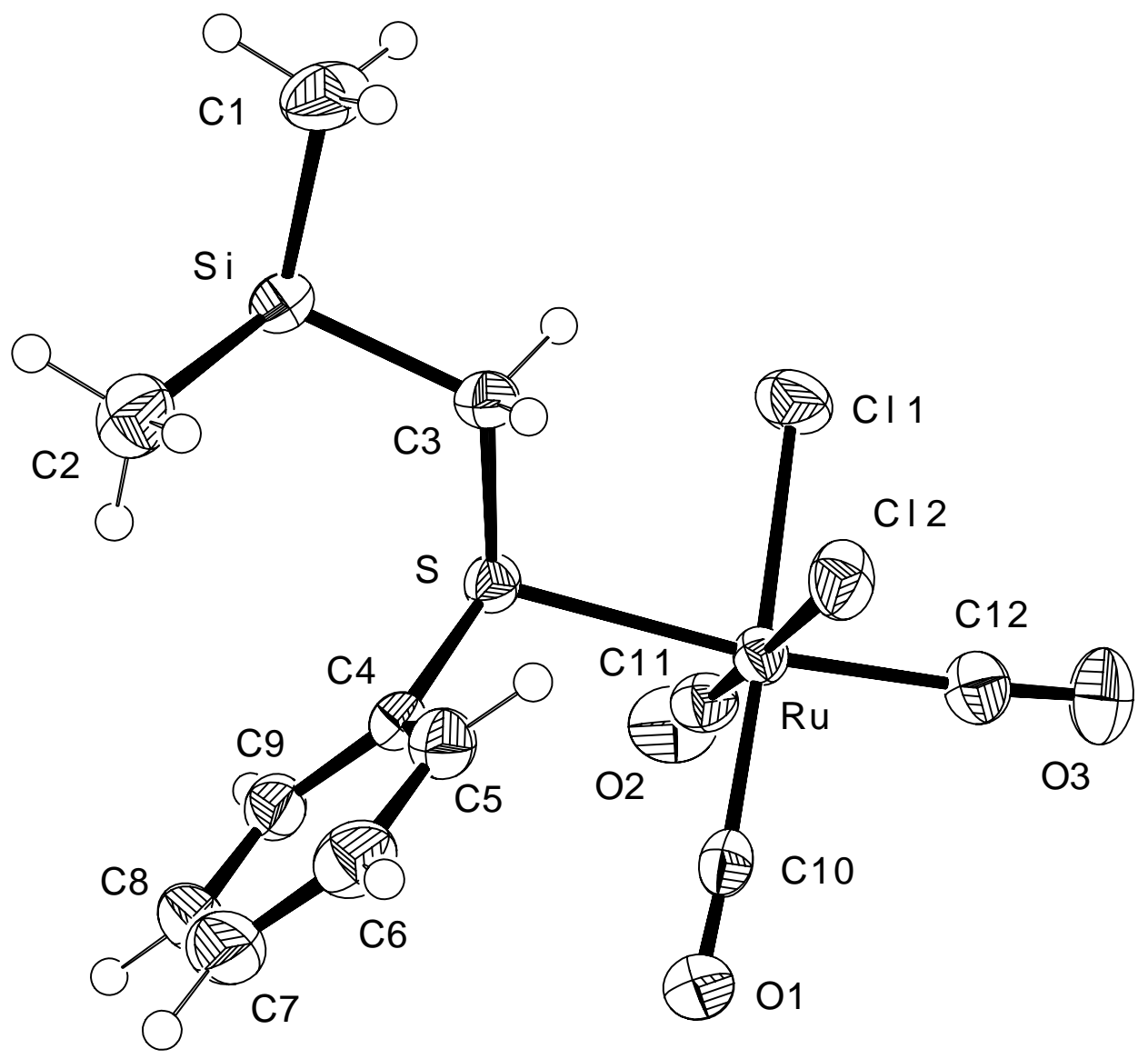


Table 26. Crystal data and structure refinement for 7.

Identification code

Empirical formula

Formula weight

Temperature

Wavelength

Crystal system

Space group

Unit cell dimensions

Volume

Z

Density (calculated)

Absorption coefficient

$\mathrm{F}(000)$

Crystal size

Theta range for data collection

Index ranges

Reflections collected

Independent reflections

Completeness to theta $=25.00^{\circ}$

Max. and min. transmission

Refinement method

Data / restraints / parameters

Goodness-of-fit on $\mathrm{F}^{2}$

Final $\mathrm{R}$ indices $[\mathrm{I}>2 \operatorname{sigma}(\mathrm{I})]$

$\mathrm{R}$ indices (all data)

Absolute structure parameter

Largest diff. peak and hole
7

C26 H32 S2 Si2

464.82

173(2) K

$0.71073 \AA$

orthorhombic

Pca2(1)

$\mathrm{a}=25.243(4) \AA$

$\alpha=90^{\circ}$.

$\mathrm{b}=6.464(2) \AA$

$\beta=90^{\circ}$.

$\mathrm{c}=31.369(6) \AA$

$\gamma=90^{\circ}$.

8

$1.206 \mathrm{Mg} / \mathrm{m}^{3}$

$0.313 \mathrm{~mm}^{-1}$

1984

$0.40 \times 0.20 \times 0.20 \mathrm{~mm}^{3}$

2.07 to $25.00^{\circ}$.

$-30<=\mathrm{h}<=28,-5<=\mathrm{k}<=7,-36<=\mathrm{l}<=31$

13649

$8206[\mathrm{R}($ int $)=0.0862]$

$98.6 \%$

0.9400 and 0.8850

Full-matrix least-squares on $\mathrm{F}^{2}$

8206 / 1 / 549

1.011

$\mathrm{R} 1=0.0617, \mathrm{wR} 2=0.1143$

$\mathrm{R} 1=0.1274, \mathrm{wR} 2=0.1291$

$0.07(17)$

0.464 and -0.323 e. $\AA^{-3}$ 
Table 27. Atomic coordinates ( $\left.\times 10^{4}\right)$ and equivalent isotropic displacement parameters $\left(\AA^{2} \times 10^{3}\right)$ for 7. $U(e q)$ is defined as one third of the trace of the orthogonalized $U^{i j}$ tensor.

\begin{tabular}{|c|c|c|c|c|}
\hline & $\mathrm{x}$ & $\mathrm{y}$ & z & $\mathrm{U}(\mathrm{eq})$ \\
\hline $\mathrm{C}(1)$ & $5958(3)$ & 4194(17) & $3685(3)$ & $52(3)$ \\
\hline$C(2)$ & $5636(3)$ & $-268(16)$ & $3438(4)$ & $54(3)$ \\
\hline$C(3)$ & $6175(3)$ & $2419(16)$ & 2798(3) & $45(2)$ \\
\hline$C(4)$ & $6672(3)$ & $4693(16)$ & $2132(4)$ & $45(3)$ \\
\hline$C(5)$ & $6785(4)$ & $6459(19)$ & 1902(3) & $58(3)$ \\
\hline$C(6)$ & $7074(4)$ & $6340(20)$ & $1529(4)$ & $66(3)$ \\
\hline$C(7)$ & $7271(4)$ & $4500(20)$ & $1390(4)$ & $58(3)$ \\
\hline$C(8)$ & $7174(3)$ & 2742(19) & $1623(4)$ & $56(3)$ \\
\hline$C(9)$ & $6866(3)$ & $2825(18)$ & 1987(3) & $48(3)$ \\
\hline$C(10)$ & $5059(3)$ & $3592(14)$ & $3003(3)$ & $40(2)$ \\
\hline$C(11)$ & $4539(3)$ & $3500(13)$ & 3091(3) & $37(2)$ \\
\hline$C(12)$ & $4186(2)$ & $4443(15)$ & $2752(3)$ & $32(2)$ \\
\hline$C(13)$ & $3761(3)$ & $3300(14)$ & 2594(3) & $42(2)$ \\
\hline$C(14)$ & $3454(3)$ & $4054(18)$ & $2266(3)$ & $52(3)$ \\
\hline$C(15)$ & $3547(3)$ & $6034(19)$ & $2102(3)$ & $48(3)$ \\
\hline$C(16)$ & $3959(3)$ & $7148(16)$ & $2264(3)$ & $48(2)$ \\
\hline$C(17)$ & $4284(3)$ & $6367(15)$ & $2579(3)$ & $43(2)$ \\
\hline$C(18)$ & $3555(3)$ & $3752(16)$ & $3658(3)$ & $51(2)$ \\
\hline C(19) & $4090(4)$ & $-467(15)$ & $3513(4)$ & $50(3)$ \\
\hline$C(20)$ & $4595(3)$ & $2678(16)$ & 4092(2) & $42(2)$ \\
\hline $\mathrm{C}(21)$ & $4885(3)$ & $5275(15)$ & $4773(3)$ & $37(2)$ \\
\hline$C(22)$ & $4838(3)$ & $3618(16)$ & $5052(3)$ & $42(2)$ \\
\hline$C(23)$ & $5011(3)$ & $3681(16)$ & $5459(3)$ & $46(2)$ \\
\hline $\mathrm{C}(24)$ & $5232(3)$ & $5480(20)$ & $5608(4)$ & $54(3)$ \\
\hline$C(25)$ & $5289(3)$ & $7200(20)$ & $5354(3)$ & $56(3)$ \\
\hline$C(26)$ & $5113(3)$ & $7110(15)$ & 4937(3) & $46(2)$ \\
\hline$C(27)$ & $8209(3)$ & $5395(16)$ & 4994(4) & $52(3)$ \\
\hline $\mathrm{C}(28)$ & $8539(3)$ & $943(16)$ & $4714(3)$ & $50(2)$ \\
\hline$C(29)$ & $8762(2)$ & $2615(15)$ & $5619(3)$ & $42(2)$ \\
\hline$C(30)$ & $9273(3)$ & $324(17)$ & $6272(4)$ & $44(3)$ \\
\hline$C(31)$ & $9453(3)$ & $2258(19)$ & $6417(3)$ & $55(3)$ \\
\hline$C(32)$ & $9741(3)$ & 2302(19) & $6803(3)$ & $54(3)$ \\
\hline$C(33)$ & $9864(3)$ & $580(20)$ & $7015(4)$ & $59(3)$ \\
\hline$C(34)$ & $9678(4)$ & $-1320(20)$ & $6869(4)$ & $61(3)$ \\
\hline
\end{tabular}




\begin{tabular}{|c|c|c|c|c|}
\hline$C(35)$ & 9392(3) & $-1452(18)$ & 6491(4) & $59(3)$ \\
\hline$C(36)$ & 7654(2) & $1526(13)$ & 5419(3) & $36(2)$ \\
\hline$C(37)$ & $7126(3)$ & $1586(13)$ & $5352(3)$ & $33(2)$ \\
\hline$C(38)$ & $6775(3)$ & 691(16) & $5678(3)$ & $38(2)$ \\
\hline $\mathrm{C}(39)$ & $6867(3)$ & $-1309(16)$ & $5834(3)$ & $43(2)$ \\
\hline$C(40)$ & $6548(3)$ & $-2131(17)$ & 6164(3) & $52(2)$ \\
\hline $\mathrm{C}(41)$ & $6122(3)$ & $-942(19)$ & $6318(3)$ & $56(3)$ \\
\hline $\mathrm{C}(42)$ & 6015(3) & $950(20)$ & 6151(3) & $55(3)$ \\
\hline$C(43)$ & 6342(3) & $1782(15)$ & $5836(3)$ & $39(2)$ \\
\hline $\mathrm{C}(44)$ & $6141(2)$ & $1304(15)$ & 4774(3) & $44(2)$ \\
\hline$C(45)$ & 6652(3) & $5546(18)$ & 4915(4) & $55(3)$ \\
\hline $\mathrm{C}(46)$ & $7186(2)$ & $2407(17)$ & $4360(3)$ & $43(2)$ \\
\hline $\mathrm{C}(47)$ & $7347(3)$ & $172(14)$ & $3599(4)$ & $39(3)$ \\
\hline $\mathrm{C}(48)$ & 7589(3) & $1886(17)$ & $3443(3)$ & $50(3)$ \\
\hline C(49) & $7746(3)$ & 1999(19) & $3018(3)$ & $58(3)$ \\
\hline$C(50)$ & $7665(3)$ & $336(19)$ & $2750(4)$ & $55(3)$ \\
\hline$C(51)$ & 7414(3) & $-1398(17)$ & 2899(3) & $55(3)$ \\
\hline$C(52)$ & $7258(3)$ & $-1512(16)$ & $3339(3)$ & $53(3)$ \\
\hline $\mathrm{S}(1)$ & $6283(1)$ & $5010(4)$ & $2598(1)$ & $58(1)$ \\
\hline$S(2)$ & $4656(1)$ & $5338(4)$ & $4243(1)$ & $49(1)$ \\
\hline $\mathrm{S}(3)$ & 8893(1) & $23(4)$ & $5798(1)$ & $55(1)$ \\
\hline $\mathrm{S}(4)$ & $7147(1)$ & $-159(4)$ & 4141(1) & $52(1)$ \\
\hline $\operatorname{Si}(1)$ & $5686(1)$ & $2473(5)$ & $3256(1)$ & $43(1)$ \\
\hline $\operatorname{Si}(2)$ & $4201(1)$ & $2367(4)$ & $3573(1)$ & $37(1)$ \\
\hline $\operatorname{Si}(3)$ & $8269(1)$ & $2597(4)$ & $5159(1)$ & $36(1)$ \\
\hline $\operatorname{Si}(4)$ & $6782(1)$ & 2704(4) & $4860(1)$ & $36(1)$ \\
\hline
\end{tabular}


Table 28. Bond lengths $[\AA]$ and angles $\left[{ }^{\circ}\right]$ for 7.

\begin{tabular}{|c|c|c|c|}
\hline $\mathrm{C}(1)-\mathrm{Si}(1)$ & $1.875(10)$ & $\mathrm{C}(30)-\mathrm{C}(31)$ & $1.406(15)$ \\
\hline $\mathrm{C}(2)-\mathrm{Si}(1)$ & $1.866(10)$ & $\mathrm{C}(30)-\mathrm{S}(3)$ & $1.782(11)$ \\
\hline $\mathrm{C}(3)-\mathrm{S}(1)$ & $1.809(10)$ & $\mathrm{C}(31)-\mathrm{C}(32)$ & $1.412(13)$ \\
\hline$C(3)-\operatorname{Si}(1)$ & $1.895(8)$ & $\mathrm{C}(32)-\mathrm{C}(33)$ & $1.335(16)$ \\
\hline$C(4)-C(5)$ & $1.379(14)$ & $\mathrm{C}(33)-\mathrm{C}(34)$ & $1.392(17)$ \\
\hline$C(4)-C(9)$ & $1.380(14)$ & $C(34)-C(35)$ & $1.390(16)$ \\
\hline$C(4)-S(1)$ & $1.774(11)$ & $C(36)-C(37)$ & $1.351(9)$ \\
\hline$C(5)-C(6)$ & $1.381(15)$ & $\mathrm{C}(36)-\mathrm{Si}(3)$ & $1.885(7)$ \\
\hline$C(6)-C(7)$ & $1.361(17)$ & $\mathrm{C}(37)-\mathrm{C}(38)$ & $1.470(12)$ \\
\hline$C(7)-C(8)$ & $1.374(16)$ & $\mathrm{C}(37)-\mathrm{Si}(4)$ & $1.914(8)$ \\
\hline $\mathrm{C}(8)-\mathrm{C}(9)$ & $1.380(14)$ & $\mathrm{C}(38)-\mathrm{C}(43)$ & $1.392(11)$ \\
\hline $\mathrm{C}(10)-\mathrm{C}(11)$ & $1.341(10)$ & $\mathrm{C}(38)-\mathrm{C}(39)$ & $1.402(13)$ \\
\hline $\mathrm{C}(10)-\mathrm{Si}(1)$ & $1.912(8)$ & $C(39)-C(40)$ & $1.415(12)$ \\
\hline $\mathrm{C}(11)-\mathrm{C}(12)$ & $1.517(11)$ & $C(40)-C(41)$ & $1.406(13)$ \\
\hline$C(11)-\operatorname{Si}(2)$ & $1.886(9)$ & $C(41)-C(42)$ & $1.360(16)$ \\
\hline$C(12)-C(17)$ & $1.379(13)$ & $C(42)-C(43)$ & $1.394(13)$ \\
\hline$C(12)-C(13)$ & $1.392(11)$ & $\mathrm{C}(44)-\operatorname{Si}(4)$ & $1.874(7)$ \\
\hline$C(13)-C(14)$ & $1.378(12)$ & $\mathrm{C}(45)-\mathrm{Si}(4)$ & $1.874(12)$ \\
\hline$C(14)-C(15)$ & $1.399(16)$ & $\mathrm{C}(46)-\mathrm{S}(4)$ & $1.798(11)$ \\
\hline$C(15)-C(16)$ & $1.362(13)$ & $\mathrm{C}(46)-\mathrm{Si}(4)$ & $1.880(8)$ \\
\hline$C(16)-C(17)$ & $1.379(12)$ & $\mathrm{C}(47)-\mathrm{C}(48)$ & $1.356(13)$ \\
\hline C(18)-Si(2) & $1.880(8)$ & $C(47)-C(52)$ & $1.378(13)$ \\
\hline C(19)-Si(2) & $1.863(10)$ & $\mathrm{C}(47)-\mathrm{S}(4)$ & $1.788(12)$ \\
\hline$C(20)-S(2)$ & $1.790(10)$ & $\mathrm{C}(48)-\mathrm{C}(49)$ & $1.393(13)$ \\
\hline$C(20)-\operatorname{Si}(2)$ & $1.919(7)$ & $C(49)-C(50)$ & $1.379(15)$ \\
\hline$C(21)-C(22)$ & $1.388(13)$ & $C(50)-C(51)$ & $1.371(15)$ \\
\hline$C(21)-C(26)$ & $1.414(13)$ & $\mathrm{C}(51)-\mathrm{C}(52)$ & $1.438(13)$ \\
\hline $\mathrm{C}(21)-\mathrm{S}(2)$ & $1.763(11)$ & & \\
\hline$C(22)-C(23)$ & $1.351(13)$ & $\mathrm{S}(1)-\mathrm{C}(3)-\mathrm{Si}(1)$ & $110.1(5)$ \\
\hline$C(23)-C(24)$ & $1.369(15)$ & $C(5)-C(4)-C(9)$ & $118.6(10)$ \\
\hline$C(24)-C(25)$ & $1.379(15)$ & $C(5)-C(4)-S(1)$ & $116.7(8)$ \\
\hline$C(25)-C(26)$ & $1.384(13)$ & $\mathrm{C}(9)-\mathrm{C}(4)-\mathrm{S}(1)$ & $124.7(8)$ \\
\hline C(27)-Si(3) & $1.887(11)$ & $C(4)-C(5)-C(6)$ & $120.3(11)$ \\
\hline C(28)-Si(3) & $1.885(10)$ & $C(7)-C(6)-C(5)$ & $121.0(11)$ \\
\hline$C(29)-S(3)$ & $1.797(9)$ & $C(6)-C(7)-C(8)$ & $119.0(11)$ \\
\hline C(29)-Si(3) & $1.907(8)$ & $C(7)-C(8)-C(9)$ & $120.6(11)$ \\
\hline $\mathrm{C}(30)-\mathrm{C}(35)$ & $1.372(14)$ & $\mathrm{C}(8)-\mathrm{C}(9)-\mathrm{C}(4)$ & $120.4(10)$ \\
\hline
\end{tabular}




\begin{tabular}{|c|c|c|c|}
\hline $\mathrm{C}(11)-\mathrm{C}(10)-\mathrm{Si}(1)$ & $135.1(7)$ & $\mathrm{C}(42)-\mathrm{C}(41)-\mathrm{C}(40)$ & $120.9(9)$ \\
\hline$C(10)-C(11)-C(12)$ & $114.5(7)$ & $\mathrm{C}(41)-\mathrm{C}(42)-\mathrm{C}(43)$ & $120.0(9)$ \\
\hline$C(10)-C(11)-\operatorname{Si}(2)$ & $128.7(6)$ & $C(38)-C(43)-C(42)$ & $121.5(10)$ \\
\hline $\mathrm{C}(12)-\mathrm{C}(11)-\mathrm{Si}(2)$ & $116.9(5)$ & $\mathrm{S}(4)-\mathrm{C}(46)-\mathrm{Si}(4)$ & $112.5(5)$ \\
\hline$C(17)-C(12)-C(13)$ & $118.5(7)$ & $\mathrm{C}(48)-\mathrm{C}(47)-\mathrm{C}(52)$ & $120.4(10)$ \\
\hline$C(17)-C(12)-C(11)$ & $122.2(7)$ & $\mathrm{C}(48)-\mathrm{C}(47)-\mathrm{S}(4)$ & $124.6(8)$ \\
\hline$C(13)-C(12)-C(11)$ & 119.3(8) & $\mathrm{C}(52)-\mathrm{C}(47)-\mathrm{S}(4)$ & $114.9(7)$ \\
\hline$C(14)-C(13)-C(12)$ & $120.7(9)$ & $\mathrm{C}(47)-\mathrm{C}(48)-\mathrm{C}(49)$ & $121.0(10)$ \\
\hline$C(13)-C(14)-C(15)$ & $120.2(8)$ & $\mathrm{C}(50)-\mathrm{C}(49)-\mathrm{C}(48)$ & $120.0(11)$ \\
\hline$C(16)-C(15)-C(14)$ & $118.4(9)$ & $\mathrm{C}(51)-\mathrm{C}(50)-\mathrm{C}(49)$ & $119.8(10)$ \\
\hline$C(15)-C(16)-C(17)$ & $121.7(10)$ & $\mathrm{C}(50)-\mathrm{C}(51)-\mathrm{C}(52)$ & $119.7(10)$ \\
\hline$C(12)-C(17)-C(16)$ & $120.4(8)$ & $\mathrm{C}(47)-\mathrm{C}(52)-\mathrm{C}(51)$ & $118.8(10)$ \\
\hline $\mathrm{S}(2)-\mathrm{C}(20)-\mathrm{Si}(2)$ & $111.6(5)$ & $\mathrm{C}(4)-\mathrm{S}(1)-\mathrm{C}(3)$ & $105.2(4)$ \\
\hline$C(22)-C(21)-C(26)$ & 117.0(9) & $C(21)-S(2)-C(20)$ & $104.8(4)$ \\
\hline $\mathrm{C}(22)-\mathrm{C}(21)-\mathrm{S}(2)$ & $125.8(7)$ & $\mathrm{C}(30)-\mathrm{S}(3)-\mathrm{C}(29)$ & $104.9(5)$ \\
\hline $\mathrm{C}(26)-\mathrm{C}(21)-\mathrm{S}(2)$ & $117.1(8)$ & $\mathrm{C}(47)-\mathrm{S}(4)-\mathrm{C}(46)$ & $103.8(4)$ \\
\hline$C(23)-C(22)-C(21)$ & $123.0(9)$ & $\mathrm{C}(2)-\mathrm{Si}(1)-\mathrm{C}(1)$ & $111.6(5)$ \\
\hline$C(22)-C(23)-C(24)$ & $118.7(10)$ & $\mathrm{C}(2)-\mathrm{Si}(1)-\mathrm{C}(3)$ & $105.0(5)$ \\
\hline$C(23)-C(24)-C(25)$ & $122.1(10)$ & $\mathrm{C}(1)-\mathrm{Si}(1)-\mathrm{C}(3)$ & $108.5(4)$ \\
\hline$C(24)-C(25)-C(26)$ & $118.6(10)$ & $\mathrm{C}(2)-\mathrm{Si}(1)-\mathrm{C}(10)$ & $115.5(4)$ \\
\hline$C(25)-C(26)-C(21)$ & $120.6(10)$ & $\mathrm{C}(1)-\mathrm{Si}(1)-\mathrm{C}(10)$ & 112.1(4) \\
\hline $\mathrm{S}(3)-\mathrm{C}(29)-\mathrm{Si}(3)$ & $110.5(5)$ & $\mathrm{C}(3)-\mathrm{Si}(1)-\mathrm{C}(10)$ & $103.4(4)$ \\
\hline$C(35)-C(30)-C(31)$ & $120.8(10)$ & $\mathrm{C}(19)-\mathrm{Si}(2)-\mathrm{C}(18)$ & $110.6(4)$ \\
\hline $\mathrm{C}(35)-\mathrm{C}(30)-\mathrm{S}(3)$ & $116.5(8)$ & $\mathrm{C}(19)-\mathrm{Si}(2)-\mathrm{C}(11)$ & $111.6(5)$ \\
\hline $\mathrm{C}(31)-\mathrm{C}(30)-\mathrm{S}(3)$ & $122.7(8)$ & $\mathrm{C}(18)-\mathrm{Si}(2)-\mathrm{C}(11)$ & $108.8(4)$ \\
\hline$C(30)-C(31)-C(32)$ & $117.4(11)$ & $\mathrm{C}(19)-\mathrm{Si}(2)-\mathrm{C}(20)$ & $105.5(5)$ \\
\hline $\mathrm{C}(33)-\mathrm{C}(32)-\mathrm{C}(31)$ & $122.0(11)$ & $\mathrm{C}(18)-\mathrm{Si}(2)-\mathrm{C}(20)$ & $106.3(4)$ \\
\hline$C(32)-C(33)-C(34)$ & $119.8(11)$ & $\mathrm{C}(11)-\mathrm{Si}(2)-\mathrm{C}(20)$ & $113.9(3)$ \\
\hline$C(35)-C(34)-C(33)$ & $120.4(11)$ & $\mathrm{C}(36)-\mathrm{Si}(3)-\mathrm{C}(28)$ & $114.2(4)$ \\
\hline $\mathrm{C}(30)-\mathrm{C}(35)-\mathrm{C}(34)$ & $119.5(11)$ & $\mathrm{C}(36)-\mathrm{Si}(3)-\mathrm{C}(27)$ & $113.9(4)$ \\
\hline $\mathrm{C}(37)-\mathrm{C}(36)-\mathrm{Si}(3)$ & $137.3(7)$ & $\mathrm{C}(28)-\mathrm{Si}(3)-\mathrm{C}(27)$ & $111.7(5)$ \\
\hline$C(36)-C(37)-C(38)$ & $118.3(7)$ & $\mathrm{C}(36)-\mathrm{Si}(3)-\mathrm{C}(29)$ & $102.2(3)$ \\
\hline $\mathrm{C}(36)-\mathrm{C}(37)-\mathrm{Si}(4)$ & $125.8(6)$ & $\mathrm{C}(28)-\mathrm{Si}(3)-\mathrm{C}(29)$ & 109.1(4) \\
\hline $\mathrm{C}(38)-\mathrm{C}(37)-\mathrm{Si}(4)$ & $115.9(5)$ & $\mathrm{C}(27)-\mathrm{Si}(3)-\mathrm{C}(29)$ & $104.7(5)$ \\
\hline $\mathrm{C}(43)-\mathrm{C}(38)-\mathrm{C}(39)$ & $118.2(8)$ & $\mathrm{C}(44)-\mathrm{Si}(4)-\mathrm{C}(45)$ & $109.6(4)$ \\
\hline $\mathrm{C}(43)-\mathrm{C}(38)-\mathrm{C}(37)$ & $121.4(9)$ & $\mathrm{C}(44)-\mathrm{Si}(4)-\mathrm{C}(46)$ & $107.4(4)$ \\
\hline $\mathrm{C}(39)-\mathrm{C}(38)-\mathrm{C}(37)$ & $120.4(7)$ & $\mathrm{C}(45)-\mathrm{Si}(4)-\mathrm{C}(46)$ & $105.8(5)$ \\
\hline$C(38)-C(39)-C(40)$ & $120.5(8)$ & $\mathrm{C}(44)-\mathrm{Si}(4)-\mathrm{C}(37)$ & $109.0(4)$ \\
\hline$C(41)-C(40)-C(39)$ & 118.7(9) & $\mathrm{C}(45)-\mathrm{Si}(4)-\mathrm{C}(37)$ & $112.0(5)$ \\
\hline
\end{tabular}


$\mathrm{C}(46)-\mathrm{Si}(4)-\mathrm{C}(37)$

$112.8(3)$

Symmetry transformations used to generate equivalent atoms: 
Table 29. Anisotropic displacement parameters $\left(\AA^{2} \times 10^{3}\right)$ for 7 . The anisotropic displacement factor exponent takes the form: $-2 \pi^{2}\left[h^{2} a^{* 2} U^{11}+\ldots+2 h k a^{*} b^{*} U^{12}\right]$

\begin{tabular}{|c|c|c|c|c|c|c|}
\hline & $\mathrm{U}^{11}$ & $\mathrm{U}^{22}$ & $\mathrm{U}^{33}$ & $\mathrm{U}^{23}$ & $\mathrm{U}^{13}$ & $\mathrm{U}^{12}$ \\
\hline $\mathrm{C}(1)$ & $60(5)$ & $54(7)$ & $43(6)$ & $4(5)$ & $-23(4)$ & $3(4)$ \\
\hline$C(2)$ & $53(4)$ & $49(7)$ & $61(8)$ & $13(6)$ & $5(5)$ & $11(4)$ \\
\hline $\mathrm{C}(3)$ & $35(3)$ & $51(6)$ & $49(6)$ & $1(5)$ & 1(3) & $9(4)$ \\
\hline$C(4)$ & $37(4)$ & $49(7)$ & $50(7)$ & $4(5)$ & $-4(4)$ & $9(4)$ \\
\hline$C(5)$ & $62(6)$ & $56(8)$ & $55(7)$ & $4(6)$ & $4(5)$ & $-2(5)$ \\
\hline$C(6)$ & $69(6)$ & $67(9)$ & $61(8)$ & $13(7)$ & $4(5)$ & $-17(6)$ \\
\hline$C(7)$ & $61(5)$ & 74(9) & $38(7)$ & $-4(7)$ & $6(4)$ & $2(6)$ \\
\hline $\mathrm{C}(8)$ & $56(5)$ & $52(7)$ & $61(7)$ & $4(6)$ & $3(5)$ & $14(5)$ \\
\hline $\mathrm{C}(9)$ & 44(4) & $54(7)$ & $45(6)$ & $20(6)$ & 1(4) & $1(5)$ \\
\hline $\mathrm{C}(10)$ & $46(4)$ & $44(5)$ & $30(5)$ & $6(4)$ & $-7(3)$ & $0(4)$ \\
\hline $\mathrm{C}(11)$ & $35(4)$ & $40(5)$ & $37(5)$ & $-7(4)$ & $-10(3)$ & $0(3)$ \\
\hline $\mathrm{C}(12)$ & $34(4)$ & $41(5)$ & $19(5)$ & $-6(4)$ & 1(3) & $5(3)$ \\
\hline$C(13)$ & $46(4)$ & $50(6)$ & $29(5)$ & $0(4)$ & $-2(3)$ & $-4(4)$ \\
\hline $\mathrm{C}(14)$ & $54(5)$ & $75(8)$ & $28(6)$ & $-3(6)$ & $-9(4)$ & $-12(5)$ \\
\hline$C(15)$ & $50(5)$ & $65(8)$ & $31(6)$ & $6(6)$ & $-5(4)$ & $13(5)$ \\
\hline$C(16)$ & $60(5)$ & $50(6)$ & $34(5)$ & $16(5)$ & $-3(4)$ & $15(5)$ \\
\hline$C(17)$ & $36(4)$ & $52(6)$ & $42(6)$ & $-10(6)$ & $6(4)$ & $-1(4)$ \\
\hline$C(18)$ & $45(4)$ & $70(7)$ & $37(6)$ & $8(5)$ & $0(4)$ & $-7(4)$ \\
\hline$C(19)$ & $65(5)$ & $46(6)$ & $40(7)$ & $9(5)$ & $-5(4)$ & $-4(5)$ \\
\hline$C(20)$ & 41(4) & $56(6)$ & $28(4)$ & $-4(5)$ & $-7(3)$ & $-1(4)$ \\
\hline $\mathrm{C}(21)$ & $25(3)$ & $52(6)$ & $32(6)$ & $3(5)$ & $5(3)$ & 7(3) \\
\hline$C(22)$ & $36(4)$ & $44(6)$ & $47(7)$ & $-16(5)$ & $-2(4)$ & $-3(4)$ \\
\hline$C(23)$ & $46(5)$ & $54(7)$ & $37(6)$ & 11(5) & $-8(4)$ & 2(4) \\
\hline$C(24)$ & $38(4)$ & $88(9)$ & $35(6)$ & $-15(6)$ & 1(4) & $2(5)$ \\
\hline$C(25)$ & $46(4)$ & $73(8)$ & $50(6)$ & $-17(6)$ & $1(4)$ & $-9(5)$ \\
\hline$C(26)$ & $46(4)$ & $38(6)$ & $55(6)$ & $-6(5)$ & $1(4)$ & $1(4)$ \\
\hline$C(27)$ & 49(4) & $53(6)$ & $53(8)$ & $6(6)$ & $4(4)$ & $-6(4)$ \\
\hline$C(28)$ & $45(4)$ & $54(6)$ & $50(6)$ & $-1(5)$ & $6(4)$ & $4(4)$ \\
\hline$C(29)$ & $32(3)$ & $45(5)$ & $49(5)$ & $13(5)$ & $5(3)$ & $-5(4)$ \\
\hline$C(30)$ & $45(4)$ & $51(7)$ & $36(6)$ & $6(5)$ & $6(4)$ & $4(4)$ \\
\hline $\mathrm{C}(31)$ & $55(5)$ & $55(7)$ & $55(7)$ & $-5(6)$ & $6(4)$ & $-3(5)$ \\
\hline $\mathrm{C}(32)$ & $53(5)$ & $66(7)$ & $44(6)$ & $-14(6)$ & $-6(4)$ & $11(5)$ \\
\hline$C(33)$ & $48(5)$ & 79(9) & $52(8)$ & $-9(7)$ & $-1(4)$ & $16(5)$ \\
\hline$C(34)$ & $63(6)$ & $68(9)$ & $53(8)$ & $10(7)$ & $6(5)$ & $16(6)$ \\
\hline
\end{tabular}




\begin{tabular}{|c|c|c|c|c|c|c|}
\hline$C(35)$ & $57(5)$ & $55(8)$ & $64(8)$ & $5(6)$ & $-8(5)$ & $-6(5)$ \\
\hline$C(36)$ & $34(4)$ & $35(5)$ & $37(5)$ & 2(4) & $0(3)$ & $0(3)$ \\
\hline$C(37)$ & $31(3)$ & $43(5)$ & $25(4)$ & $8(4)$ & $2(3)$ & $3(3)$ \\
\hline$C(38)$ & $31(4)$ & $45(6)$ & $36(6)$ & $6(5)$ & $-3(3)$ & $-3(4)$ \\
\hline$C(39)$ & 41(4) & $57(6)$ & $32(5)$ & $2(5)$ & $4(4)$ & $-1(4)$ \\
\hline $\mathrm{C}(40)$ & $57(5)$ & $60(7)$ & $38(6)$ & $4(5)$ & $5(4)$ & $-3(5)$ \\
\hline$C(41)$ & $52(5)$ & $86(9)$ & $28(6)$ & $4(6)$ & $6(4)$ & $-15(5)$ \\
\hline $\mathrm{C}(42)$ & $25(4)$ & $89(9)$ & $49(7)$ & $0(7)$ & $4(4)$ & $-3(4)$ \\
\hline$C(43)$ & $43(4)$ & $47(6)$ & $27(5)$ & $-4(4)$ & $-3(4)$ & $-8(4)$ \\
\hline$C(44)$ & $35(4)$ & $63(6)$ & $32(5)$ & $-3(5)$ & $2(3)$ & $-7(4)$ \\
\hline$C(45)$ & 49(4) & $76(7)$ & $40(7)$ & $-4(6)$ & $4(4)$ & $21(5)$ \\
\hline$C(46)$ & $39(4)$ & $48(5)$ & $42(5)$ & $8(5)$ & $-3(3)$ & $-2(4)$ \\
\hline $\mathrm{C}(47)$ & $36(4)$ & $47(7)$ & $34(6)$ & $-9(5)$ & $2(3)$ & $-8(4)$ \\
\hline $\mathrm{C}(48)$ & $44(4)$ & $66(8)$ & $40(6)$ & $-14(5)$ & $7(4)$ & $-12(4)$ \\
\hline$C(49)$ & $52(5)$ & $70(9)$ & $53(7)$ & $3(6)$ & 11(4) & $0(5)$ \\
\hline$C(50)$ & $43(4)$ & $87(9)$ & $35(6)$ & $-9(6)$ & $4(4)$ & $-4(5)$ \\
\hline$C(51)$ & $43(4)$ & $74(8)$ & $48(6)$ & $-11(6)$ & $-11(4)$ & $15(5)$ \\
\hline$C(52)$ & $43(4)$ & $68(7)$ & $48(7)$ & $-5(6)$ & $3(4)$ & $2(4)$ \\
\hline$S(1)$ & $60(1)$ & $50(2)$ & $64(2)$ & $-2(1)$ & $22(1)$ & $-3(1)$ \\
\hline$S(2)$ & $58(1)$ & $49(2)$ & $39(2)$ & $-2(1)$ & $-7(1)$ & $0(1)$ \\
\hline$S(3)$ & $57(1)$ & $45(2)$ & $64(2)$ & $1(1)$ & $-19(1)$ & $4(1)$ \\
\hline$S(4)$ & $70(1)$ & $48(2)$ & $38(2)$ & 1(1) & $16(1)$ & $-10(1)$ \\
\hline $\operatorname{Si}(1)$ & $34(1)$ & $47(2)$ & $47(1)$ & $5(2)$ & $-3(1)$ & $2(1)$ \\
\hline $\operatorname{Si}(2)$ & $41(1)$ & $38(1)$ & $31(1)$ & $3(1)$ & $-2(1)$ & $-4(1)$ \\
\hline $\operatorname{Si}(3)$ & $33(1)$ & $38(1)$ & $38(1)$ & $2(1)$ & $4(1)$ & $-3(1)$ \\
\hline $\operatorname{Si}(4)$ & $35(1)$ & $43(1)$ & $31(1)$ & $2(1)$ & $2(1)$ & $1(1)$ \\
\hline
\end{tabular}


Table 30. Hydrogen coordinates $\left(\mathrm{x} 10^{4}\right)$ and isotropic displacement parameters $\left(\AA^{2} \times 10^{3}\right)$ for 7 .

\begin{tabular}{|c|c|c|c|c|}
\hline & $\mathrm{x}$ & $\mathrm{y}$ & $\mathrm{z}$ & $\mathrm{U}(\mathrm{eq})$ \\
\hline $\mathrm{H}(1 \mathrm{~A})$ & 6082 & 5491 & 3558 & 78 \\
\hline $\mathrm{H}(1 \mathrm{~B})$ & 6254 & 3497 & 3827 & 78 \\
\hline $\mathrm{H}(1 \mathrm{C})$ & 5679 & 4492 & 3893 & 78 \\
\hline $\mathrm{H}(2 \mathrm{~A})$ & 5308 & -456 & 3600 & 82 \\
\hline $\mathrm{H}(2 \mathrm{~B})$ & 5940 & -597 & 3621 & 82 \\
\hline $\mathrm{H}(2 \mathrm{C})$ & 5636 & -1189 & 3190 & 82 \\
\hline $\mathrm{H}(3 \mathrm{~A})$ & 6515 & 1826 & 2896 & 54 \\
\hline $\mathrm{H}(3 \mathrm{~B})$ & 6036 & 1530 & 2566 & 54 \\
\hline $\mathrm{H}(5)$ & 6664 & 7765 & 2001 & 69 \\
\hline $\mathrm{H}(6)$ & 7136 & 7556 & 1368 & 79 \\
\hline $\mathrm{H}(7)$ & 7472 & 4429 & 1134 & 69 \\
\hline $\mathrm{H}(8)$ & 7319 & 1458 & 1534 & 68 \\
\hline $\mathrm{H}(9)$ & 6788 & 1590 & 2138 & 57 \\
\hline $\mathrm{H}(10)$ & 5133 & 4404 & 2758 & 48 \\
\hline $\mathrm{H}(13)$ & 3683 & 1986 & 2714 & 50 \\
\hline $\mathrm{H}(14)$ & 3179 & 3226 & 2150 & 63 \\
\hline $\mathrm{H}(15)$ & 3328 & 6589 & 1884 & 58 \\
\hline $\mathrm{H}(16)$ & 4025 & 8498 & 2156 & 58 \\
\hline $\mathrm{H}(17)$ & 4576 & 7159 & 2677 & 52 \\
\hline $\mathrm{H}(18 \mathrm{~A})$ & 3390 & 3261 & 3923 & 76 \\
\hline $\mathrm{H}(18 \mathrm{~B})$ & 3318 & 3473 & 3418 & 76 \\
\hline $\mathrm{H}(18 \mathrm{C})$ & 3619 & 5243 & 3679 & 76 \\
\hline $\mathrm{H}(19 \mathrm{~A})$ & 3894 & -987 & 3761 & 75 \\
\hline H(19B) & 4432 & -1175 & 3493 & 75 \\
\hline $\mathrm{H}(19 \mathrm{C})$ & 3884 & -728 & 3254 & 75 \\
\hline $\mathrm{H}(20 \mathrm{~A})$ & 4416 & 1906 & 4323 & 50 \\
\hline $\mathrm{H}(20 \mathrm{~B})$ & 4953 & 2077 & 4054 & 50 \\
\hline $\mathrm{H}(22)$ & 4676 & 2383 & 4952 & 51 \\
\hline $\mathrm{H}(23)$ & 4980 & 2505 & 5639 & 55 \\
\hline $\mathrm{H}(24)$ & 5351 & 5532 & 5895 & 65 \\
\hline $\mathrm{H}(25)$ & 5445 & 8429 & 5463 & 67 \\
\hline $\mathrm{H}(26)$ & 5145 & 8291 & 4758 & 56 \\
\hline $\mathrm{H}(27 \mathrm{~A})$ & 8559 & 5933 & 4920 & 77 \\
\hline
\end{tabular}




\begin{tabular}{|c|c|c|c|c|}
\hline $\mathrm{H}(27 \mathrm{~B})$ & 8062 & 6202 & 5231 & 77 \\
\hline $\mathrm{H}(27 \mathrm{C})$ & 7973 & 5503 & 4747 & 77 \\
\hline $\mathrm{H}(28 \mathrm{~A})$ & 8883 & 1491 & 4622 & 74 \\
\hline $\mathrm{H}(28 \mathrm{~B})$ & 8292 & 957 & 4474 & 74 \\
\hline $\mathrm{H}(28 \mathrm{C})$ & 8584 & -480 & 4816 & 74 \\
\hline $\mathrm{H}(29 \mathrm{~A})$ & 9097 & 3270 & 5525 & 50 \\
\hline $\mathrm{H}(29 \mathrm{~B})$ & 8617 & 3439 & 5858 & 50 \\
\hline $\mathrm{H}(31)$ & 9384 & 3489 & 6261 & 66 \\
\hline $\mathrm{H}(32)$ & 9850 & 3598 & 6915 & 65 \\
\hline H(33) & 10077 & 646 & 7264 & 71 \\
\hline H(34) & 9748 & -2543 & 7029 & 74 \\
\hline H(35) & 9280 & -2759 & 6386 & 70 \\
\hline H(36) & 7739 & 727 & 5664 & 43 \\
\hline H(39) & 7146 & -2119 & 5718 & 52 \\
\hline $\mathrm{H}(40)$ & 6621 & -3459 & 6280 & 62 \\
\hline $\mathrm{H}(41)$ & 5907 & -1471 & 6541 & 67 \\
\hline $\mathrm{H}(42)$ & 5717 & 1714 & 6249 & 65 \\
\hline H(43) & 6267 & 3121 & 5727 & 47 \\
\hline $\mathrm{H}(44 \mathrm{~A})$ & 5885 & 1731 & 4991 & 66 \\
\hline $\mathrm{H}(44 \mathrm{~B})$ & 6200 & -190 & 4794 & 66 \\
\hline $\mathrm{H}(44 \mathrm{C})$ & 6001 & 1641 & 4490 & 66 \\
\hline $\mathrm{H}(45 \mathrm{~A})$ & 6988 & 6273 & 4961 & 82 \\
\hline $\mathrm{H}(45 \mathrm{~B})$ & 6418 & 5786 & 5160 & 82 \\
\hline $\mathrm{H}(45 \mathrm{C})$ & 6483 & 6064 & 4655 & 82 \\
\hline $\mathrm{H}(46 \mathrm{~A})$ & 7060 & 3413 & 4145 & 51 \\
\hline $\mathrm{H}(46 \mathrm{~B})$ & 7561 & 2733 & 4425 & 51 \\
\hline H(48) & 7653 & 3027 & 3627 & 60 \\
\hline H(49) & 7907 & 3223 & 2912 & 70 \\
\hline $\mathrm{H}(50)$ & 7784 & 393 & 2463 & 66 \\
\hline $\mathrm{H}(51)$ & 7342 & -2520 & 2712 & 66 \\
\hline $\mathrm{H}(52)$ & 7097 & -2728 & 3449 & 63 \\
\hline
\end{tabular}


ORTEP Plot for 7 (50\% probability ellipsoids).

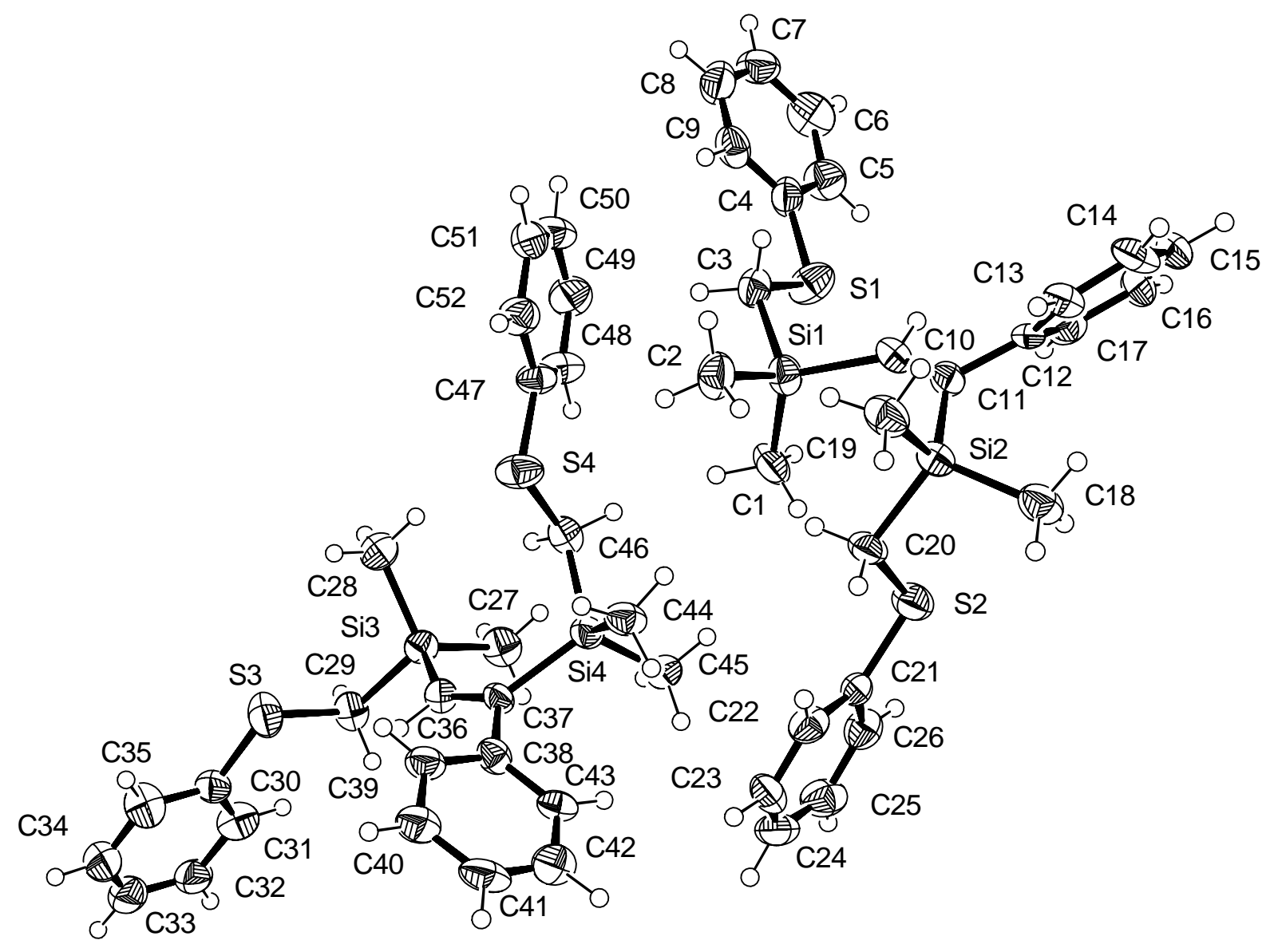




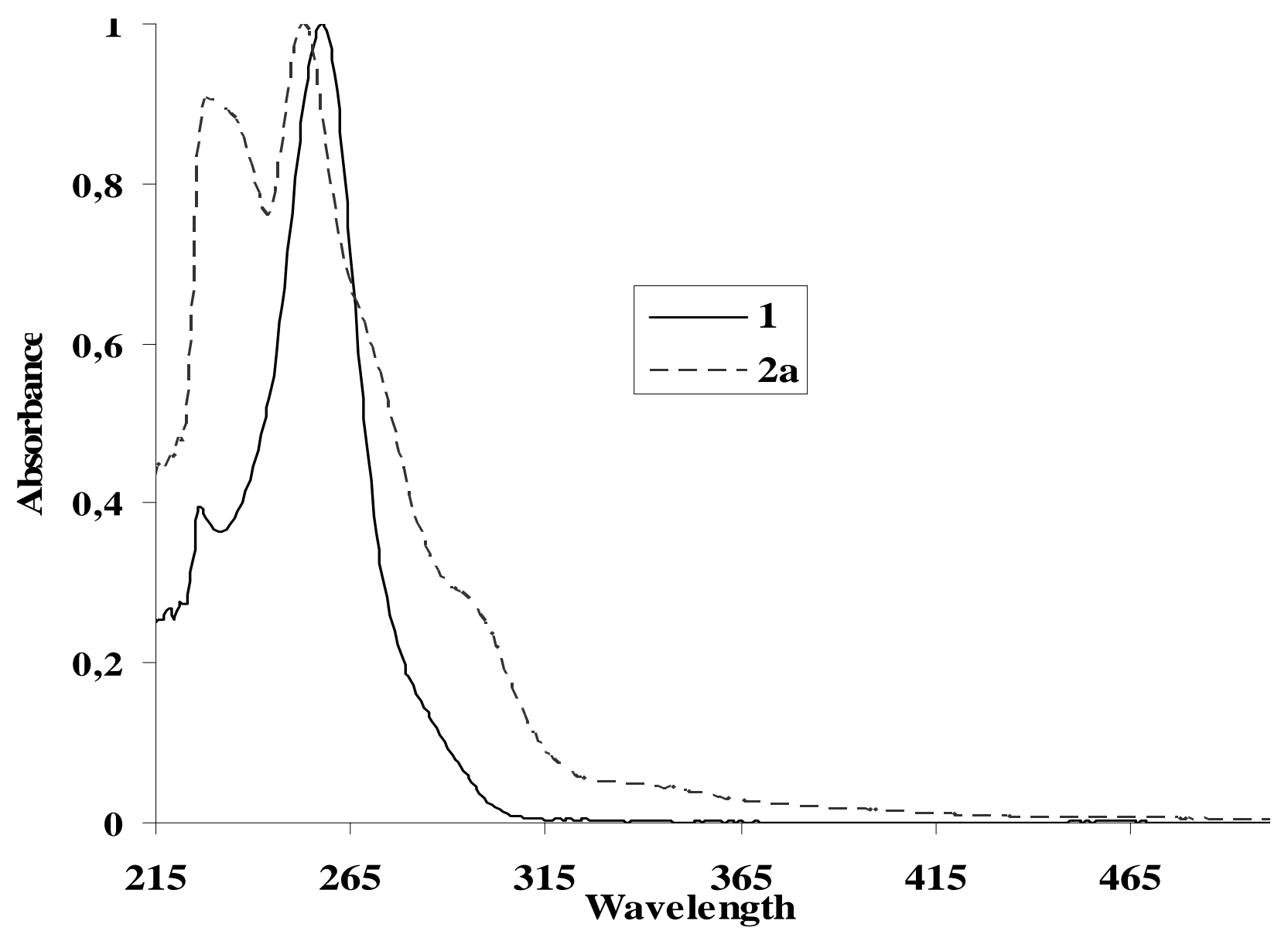

Absorption spectra of $\mathbf{1}$ and $\mathbf{2 a}$ carried out in $\mathrm{CH}_{2} \mathrm{Cl}_{2}$ at $298 \mathrm{~K}$.

\begin{tabular}{|l|l|l|}
\hline Compounds & $\mathbf{1}$ & 2a \\
\hline$\lambda_{\max }(\mathrm{nm})\left[\varepsilon\left(\mathrm{M}^{-1} \mathrm{~cm}^{-1}\right)\right]$ & $226[8000]$ & $228[25000]$ \\
& $258[20000]$ & $253[28000]$ \\
\hline
\end{tabular}




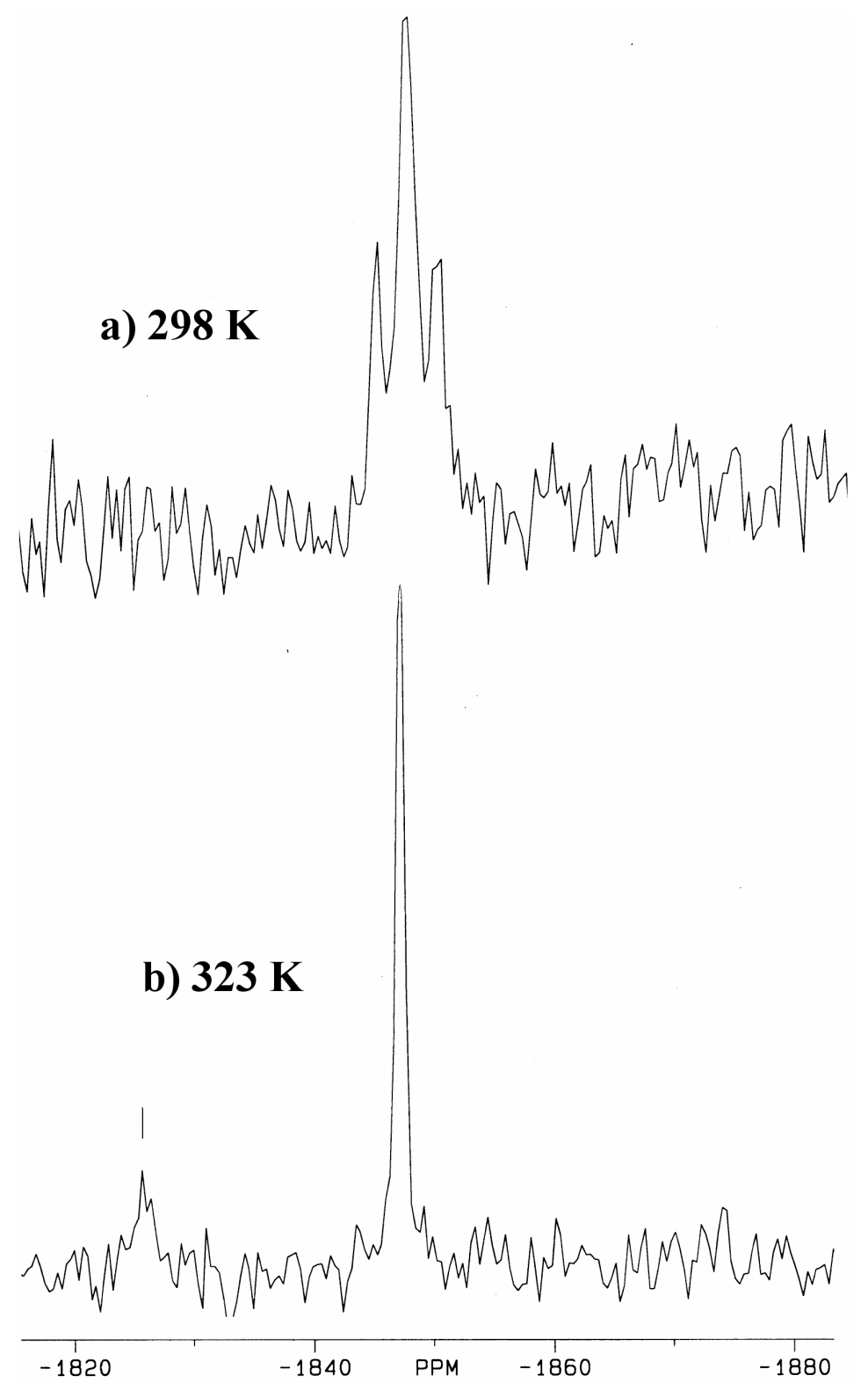

${ }^{195} \mathrm{Pt}\left\{{ }^{1} \mathrm{H}\right\}$ NMR spectrum of 2a in $\mathrm{CDCl}_{3}$ recorded at 298 and $323 \mathrm{~K}$. 